\title{
Black holes in a box: Toward the numerical evolution of black holes in AdS space-times
}

\author{
Helvi Witek, ${ }^{1, *}$ Vitor Cardoso, ${ }^{1,2, \dagger}$ Carlos Herdeiro, ${ }^{3,4, \$}$ Andrea Nerozzi, ${ }^{1, \|}$ Ulrich Sperhake, ${ }^{2,5,6, \pi}$ and Miguel Zilhão ${ }^{3, \S}$ \\ ${ }^{1}$ Centro Multidisciplinar de Astrofísica-CENTRA, Departamento de Física, Instituto Superior Técnico, \\ Universidade Técnica de Lisboa-UTL, Avenida Rovisco Pais 1, 1049-001 Lisboa, Portugal \\ ${ }^{2}$ Department of Physics and Astronomy, The University of Mississippi, University, Mississippi 38677-1848, USA \\ ${ }^{3}$ Centro de Física do Porto-CFP, Departamento de Física e Astronomia, Faculdade de Ciências da Universidade do Porto-FCUP, \\ Rua do Campo Alegre, 4169-007 Porto, Portugal \\ ${ }^{4}$ Deparamento de Física da Universidade de Aveiro, Campus de Santiago, 3810-183 Aveiro, Portugal \\ ${ }^{5}$ Institut de Ciències de l'Espai (CSIC-IEEC), Facultat de Ciències, Campus Universitat Autònoma de Barcelona, \\ E-08193 Bellaterra, Spain \\ ${ }^{6}$ California Institute of Technology, Pasadena, California 91125, USA
}

(Received 30 April 2010; published 18 November 2010)

\begin{abstract}
The evolution of black holes in "confining boxes" is interesting for a number of reasons, particularly because it mimics the global structure of anti-de Sitter geometries. These are nonglobally hyperbolic space-times and the Cauchy problem may only be well defined if the initial data are supplemented by boundary conditions at the timelike conformal boundary. Here, we explore the active role that boundary conditions play in the evolution of a bulk black hole system, by imprisoning a black hole binary in a box with mirrorlike boundary conditions. We are able to follow the post-merger dynamics for up to two reflections off the boundary of the gravitational radiation produced in the merger. We estimate that about $15 \%$ of the radiation energy is absorbed by the black hole per interaction, whereas transfer of angular momentum from the radiation to the black hole is observed only in the first interaction. We discuss the possible role of superradiant scattering for this result. Unlike the studies with outgoing boundary conditions, both of the Newman-Penrose scalars $\Psi_{4}$ and $\Psi_{0}$ are nontrivial in our setup, and we show that the numerical data verifies the expected relations between them.
\end{abstract}

DOI: 10.1103/PhysRevD.82.104037

\section{INTRODUCTION}

Numerical relativity and the gauge/gravity correspondence are two fields in high energy/gravitational physics that have seen tremendous activity and progress over the last few years. Since the 2005 breakthroughs [1-3], the numerical relativity community has produced stable evolutions of black hole $(\mathrm{BH})$ binaries in four-dimensional, asymptotically flat space-times, covering the inspiral, merger and ring-down phases. State of the art simulations can be as long as 15 orbits plus merger and ring-down [4], can deal with large mass ratios [5-8], with dimensionless BH spins of up to 0.92 [9] or with eccentricities as low as $5 \times 10^{-5}$ (see [10] for a recent review). Such simulations have unveiled new phenomena such as BH kicks [11-13] and some groups are now focusing on the construction of sufficiently accurate template waveforms to be used in filtering data from the current (LIGO, Virgo, GEO, TAMA) and planned (Advanced LIGO and LISA) gravitational wave observatories [14-17]. Simultaneously, high energy collisions of BHs have been simulated [18-20], leading to

\footnotetext{
*helvi.witek@ist.utl.pt

†vitor.cardoso@ist.utl.pt

*herdeiro@ua.pt

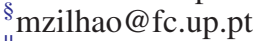

"andrea.nerozzi@ist.utl.pt

II sperhake@tapir.caltech.edu
}

PACS numbers: 04.25.D-, 04.25.dc, 04.25.dg, 04.50.-h

accurate results for the scattering cross section and for the conversion rate of the initial center-of-mass energy into gravitational radiation. These simulations have also tested cosmic censorship and the Dyson luminosity limit and exhibited the zoom-whirl behavior first found in [21].

The gauge/gravity correspondence has been developed, since the original proposal by Maldacena in 1998 [22], both as an efficient tool to understand strongly coupled gauge theories using classical gravity and as way to study gravitational phenomena from a dual field theory. Central to many of these developments are BHs, as may be seen by the following list of examples: (i) The successful microscopic computations of the Bekenstein-Hawking entropy for extremal BHs [23] and Hawking emission rates for near extremal BHs [24] are now seen as applications of the correspondence; (ii) The confinement/deconfinement phase transition in QCD-like theories has been identified [25] with the Hawking-Page phase transition for anti-de Sitter (AdS) BHs [26]; (iii) Moving away from thermal equilibrium, the quasinormal frequencies of AdS BHs have been identified with the poles of retarded correlators describing the relaxation back to equilibrium of a perturbed dual field theory [27,28]; (iv) In a large class of gauge theories with a gravity dual, a universal behavior was obtained for the ratio of the strongly coupled medium's viscosity to entropy density, by computing the absorption cross section of low energy gravitons in the dual $\mathrm{BH}$ (or black brane) geometry 
[29]. The result is in good agreement with experimental results from the Relativistic Heavy Ion Collider (RHIC); (v) Critical exponents, of the type found in spherical gravitational collapse by Choptuik in four-dimensional asymptotically flat space-time [30], have been conjectured to be dual to the asymptotic value of the parton saturation exponent for high energy scattering in QCD, in the Regge limit, at weak coupling [31]. These and other examples have built expectations that the gauge/gravity correspondence will be a useful laboratory for gaining understanding of very difficult problems of both field theory and gravity, such as the confinement problem, the information loss paradox and the problem of singularities.

Given the potential of the correspondence exemplified above, a working framework to solve Einstein's equations exactly for a broad range of initial conditions in AdS spaces would, unquestionably, be very useful for deepening the study of the correspondence, particularly in more dynamical situations. To assemble such a framework is our long-term goal and the present paper serves as the first step in this direction, i.e., to generalize the techniques of numerical relativity to AdS space-times. In order to achieve this goal, one has to go beyond the standard methods of numerical relativity in at least two obvious points.

First, AdS space-times are not globally hyperbolic. In asymptotically AdS spaces the boundary plays an "active role" for the bulk evolution. This is easily visualized in the Penrose diagram of AdS, which has a timelike boundary. Physically, null geodesics in AdS reach the boundary for a finite affine parameter. One thus often refers to an asymptotically AdS space as a "box," having in mind that AdS boundary conditions directly affect the bulk physics [32-34]. This should be contrasted with the asymptotically flat case, where the only physically relevant choice for the boundary conditions of the bulk fluctuations corresponds to outgoing waves at spatial infinity. In the gauge/gravity correspondence, the choice of the AdS boundary conditions is dictated by a holographic prescription [35-38]. Second, from the viewpoint of the duality, $D$-dimensional AdS space-times, $\mathrm{AdS}_{D}$ (not just $\mathrm{AdS}_{4}$ ) are relevant. Thus, we would like to have a framework that could be used in $\mathrm{AdS}_{D}$, in particular, for $D=5$, which is related by the correspondence to four-dimensional gauge theories. The latter issue has been recently addressed by our group in separate publications [39,40], as well as by other groups [41-46]. Here we shall focus on the former issue: the active role of boundary conditions.

The dynamics of BHs in AdS, and especially the role of spatial infinity ("the box") is poorly understood. In contrast to the asymptotically flat case, interesting new phenomena may occur in AdS backgrounds. For instance, superradiance effects have been shown to make small (as measured by the AdS radius) rotating BHs unstable, through a sequence of reflections at the boundary and amplifications close to the ergoregion [47-55]. The final state of this instability could be a new nonaxisymmetric BH configuration, which is also supported by recent gravity/hydrodynamics arguments [56,57]. Notice that nonaxisymmetric BHs are strictly forbidden in asymptotically flat space-times [58,59], so the boundary does have an important role in the description of BHs.

In order to identify in the cleanest possible way the active role of the boundary for the bulk evolution, we consider here a toy model for AdS. We set the cosmological constant to zero and impose mirrorlike boundary conditions on a box that contains the dynamical system. This mimics the AdS global geometry, keeping the local geometry of vacuum models. We choose the dynamical system to be a BH binary, starting at some given distance, producing either a head-on collision or an inspiralling merger. In the latter situation we consider the initial BHs without intrinsic angular momentum. These are, by now, very well tested systems when purely outgoing boundary conditions are imposed. Thus we will be able to see clearly the modifications due to the nonoutgoing boundary conditions in systems with nontrivial dynamics.

For the post-merger dynamics, the inspiralling binaries provide a more interesting analysis than the head-on collision case, since the initial center-of-mass energy transferred into gravitational radiation is by more than 1 order of magnitude larger in the former case as compared to the latter. Immediately after the merger, the system will contain a single (spinning or nonspinning) $\mathrm{BH}$ plus gravitational radiation. This radiation will then be (repeatedly) reflected off the boundary and interact with the $\mathrm{BH}$.

The first nontrivial result is that we can follow the numerical evolution for up to two reflections off the boundary of the gravitational radiation produced in the merger. A priori it was not guaranteed that this could be achieved, since it is not known whether the formulation of the Einstein equations that we use provides a well-defined initial value boundary problem together with the boundary conditions we impose. Our simple setting actually provides a first attempt to test the well-posedness of the initial boundary value problem in a nonglobally hyperbolic space-time. We find that our numerical results are at least second-order convergent for at least two reflections off the wall, after which we gradually lose convergence. A deeper study of these issues is clearly needed, as well as an exploration of how the convergence (and remaining results) change for different boundary conditions.

During the window of numerical convergence, we study the properties of both outgoing and ingoing gravitational radiation. The usual studies of $\mathrm{BH}$ binaries with outgoing boundary conditions focus only on the Weyl scalar $\Psi_{4}$, which describes outgoing gravitational waves. However, an equally relevant quantity for the description of gravitational radiation is the scalar $\Psi_{0}$, which describes ingoing waves but which is seldom discussed in the literature. Because of our special boundary conditions and setup, 
we are able to verify certain relations between these two quantities in a numerical evolution for the first time. This also provides a test on the correctness and meaning of the boundary conditions we have imposed.

By analyzing the properties of the apparent horizon of the $\mathrm{BH}$ produced in the merger and after each interaction with the gravitational wave packet, we estimate the amount of energy and angular momentum that is transferred from the radiation into the $\mathrm{BH}$ per interaction. In case of the inspiralling binary, the boxed $\mathrm{BH}$ is spinning and we expect superradiant scattering of the waves generated during merger to become important; in fact, the back and forth bouncing of the waves at the reflecting wall and their subsequent amplification by superradiance close to the ergoregion are expected to turn the system into a BH bomb $[48,49,60] .{ }^{1}$ Thus, these simulations will be the first attempt at a nonlinear study of the $\mathrm{BH}$ bomb. An important open problem is understanding how the evolution proceeds and what is the end point of the instability. This can only be achieved through nonlinear studies. A final statement on this issue will, however, require further analysis than that provided herein.

This paper is organized as follows. Sec. II briefly reviews the setup to evolve Einstein's equations numerically for the case at hand, including a brief description of the numerical code, of the formulation of Einstein's equations in the so-called Baumgarte-Shapiro-Shibata-Nakamura (BSSN) form, of the gauge choice adopted, of the way the "spherical" boundary is imposed and of the boundary conditions. In Sec. III we explain which of the numerical outputs we use to extract relevant physical quantities, in particular, gravitational wave estimates (Sec. III A) and apparent horizon (AH) estimates (Sec. III B). The numerical results of our simulations are shown in Sec. IV. In Sec. V we close with some discussion of the results and prospects for the future. Some technical points and further results have been organized into three appendixes. For self-containedness, Appendix A reviews the electromagnetic decomposition of the Weyl tensor and, in particular, the construction of the relevant quantities for our study, $\Psi_{0}$ and $\Psi_{4}$. Appendix B exhibits some snapshots for visualizing the evolution of the system we have studied. Appendix C describes the simulations with a cubic, rather than spherical, box.

\section{NUMERICAL FRAMEWORK}

In order to numerically generate a solution to the Einstein field equations, it is most convenient to view the problem as a time evolution or initial value problem.

\footnotetext{
${ }^{1}$ The artificial mirror sometimes appears naturally. A massive scalar field scattering off a Kerr $\mathrm{BH}$ acts as its own reflecting wall [61-67]. Furthermore, Kaluza-Klein modes in dimensional reduction can also act as an effective mass, rendering higherdimensional, rotating BHs unstable $[68,69]$. Finally, it has been suggested that astrophysical BHs might sometimes behave as $\mathrm{BH}$ bombs, with the role of the reflecting cavity being played by accretion disks [70,71].
}

The majority of formulations of the Einstein equations as an evolution system in time is based on the canonical "3 + 1"-decomposition introduced by Arnowitt, Deser and Misner (ADM) [72] and further developed by York [73]. One thus obtains a first-order, constrained evolution system in time for six components each of the three-metric $\gamma_{i j}$ and the extrinsic curvature $K_{i j}$ which describe the intrinsic geometry of three-dimensional hypersurfaces as well as their embedding in the four-dimensional spacetime. The Hamiltonian and momentum constraints impose four conditions on $\gamma_{i j}$ and $K_{i j}$ on each hypersurface but are conserved under the time evolution. Finally, four gauge variables, the lapse $\alpha$ and the shift $\beta^{i}$ represent the coordinate freedom of Einstein's relativity. Suitable specification of these free variables is crucial for a successful numerical implementation.

Our numerical framework is based on a method now commonly referred to as moving punctures [2,3]. The Einstein equations are formulated as the BSSN system $[74,75]$, a modification of the ADM formulation which employs the variables

$\chi=\psi^{-4}=\gamma^{1 / 3}, \quad \tilde{\gamma}_{i j}=\chi \gamma_{i j}$,

$K=\gamma^{i j} K_{i j}, \quad \tilde{A}_{i j}=\chi A_{i j}=\chi\left(K_{i j}-\frac{1}{3} \gamma_{i j} K\right)$,

$\tilde{\Gamma}^{i}=\tilde{\gamma}^{j k} \tilde{\Gamma}_{j k}^{i}=-\partial_{j} \tilde{\gamma}^{i j}$

By construction, $\operatorname{det} \tilde{\gamma}_{i j}=1$ which implies the last equality for $\tilde{\Gamma}^{i}$. The exact form of the evolution equations for this set of variables is given in Eqs. (A1, A4, A6, A7, A8) ${ }^{2}$ of Ref. [76]. Finally, we evolve the gauge variables $\alpha$ and $\beta^{i}$ using " $1+\log$ " slicing and a $\Gamma$-driver of the form

$$
\begin{gathered}
\partial_{t} \alpha=\beta^{m} \partial_{m} \alpha-2 \alpha K, \\
\partial_{t} \beta^{i}=\chi_{\beta} B^{i}, \\
\partial_{t} B^{i}=\partial_{t} \tilde{\Gamma}^{i}-\eta_{\beta} B^{i} .
\end{gathered}
$$

Here $\chi_{\beta}$ and $\eta_{\beta}$ are constant parameters set to one for all simulations reported in this work.

We evolve these equations with the LEAN code [76] which is based on the CACTUS computational toolkit [77] and the CARPET mesh refinement package $[78,79]$. BH binary initial data are provided by the spectral solver of Ansorg et al. [80] and the calculation of apparent horizons is performed with Thornburg's AHFINDERDIRECT [81,82]. For more details on the code, we refer the reader to Ref. [76] and Sec. III of Ref. [83].

The key ingredient in which our current numerical framework differs from previous implementations of the LEAN code and most other codes is the outer boundary

\footnotetext{
${ }^{2}$ Note that the final term on the right-hand side of their Eq. (A6) should be $\frac{2}{3} \chi\left(\alpha K-\partial_{m} \beta^{m}\right)$, i.e., a factor of $\chi$ is missing.
} 
condition, which we will discuss in more detail in the remainder of this section.

The vast majority of numerical simulations of $\mathrm{BH}$ binaries has been concerned with asymptotically flat space-times and consequently employed either of the following boundary treatments: (i) outgoing Sommerfeld conditions on Cartesian grids of finite size, as described, for example, in [84]; (ii) outgoing radiation [85,86] with multipatch methods, including Cauchy characteristic wave extraction $[87,88]$ and (iii) constraint preserving boundary conditions combined with multidomain methods $[89,90]$.

In contrast we will study the dynamics of $\mathrm{BH}$ spacetimes under the influence of a reflective outer boundary. It is natural to use for this purpose an outer boundary of spherical shape. Most importantly, this avoids mixing of different gravitational wave multipoles as would occur in the case of a reflective cubic outer boundary. This is discussed in more detail in Appendix $\mathrm{C}$ where we compare simulations using both types of boundary. Except for this comparison, however, we will exclusively study spherical outer boundaries or, rather, approximate these by using so-called Lego spheres; cf. Sec. 3 in Ref. [91]. In Fig. 1, we sketch the foliation of the space-time under consideration, suppressing one spatial dimension for simplicity. The numerical domain, i.e., the Lego sphere, is visualized by a dark (red) domain on each timeslice $\Sigma_{t+n \delta t}$. The numerical implementation of the Lego sphere is illustrated in Fig. 2, which schematically displays a computational domain using four refinement levels with one or two components each. The individual components are labeled $G_{m}^{i}$ where the indices $i$ and $m$ denote the refinement level and component number. Note that one spatial dimension is suppressed for visualization purposes. In order to update a grid function at a particular vertex, we require information from neighboring points because of the discretization of spatial derivatives in the evolution equations. The exact number $n$ of neighboring points required in each direction depends on the finite difference stencils employed. While $n=3$ for the 4 th order accurate stencils used in our simulations, we use $n=1$ for simplicity in our illustration in Fig. 2. Consider

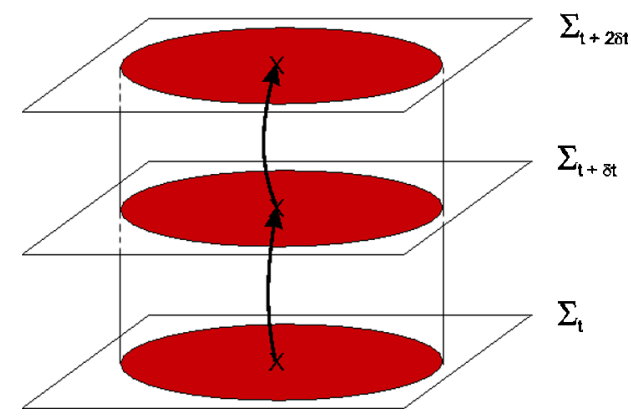

FIG. 1 (color online). Sketch of the foliation for the numerical evolution of $\mathrm{BH}$ binaries in a (spherical) box. The location of the considered numerical domain on each spatial hypersurface is shown as a dark (red) disc. first the dark (blue) shaded area inside the inner solid circle of radius $R_{B}$. Each point in this regular domain can be updated straightforwardly provided we also have valid data on the boundary points marked by $\times$ symbols. Points outside the circle of radius $R_{B}$ are not required for updating regular points and are simply ignored in the numerical evolution. The specific boundary condition is then determined by the manner in which we update grid functions on the boundary points marked as $X$ in the figure.

In order to mimic the global structure of an anti-de Sitter space-time, we effectively enclose the BH binary inside a spherical mirror and set

$$
\frac{\partial}{\partial t} f=0
$$

at each boundary point with $f$ denoting any of the BSSN variables listed in Eq. (1). The use of fourth-order stencils adds one complication to this picture: the upgrade of a grid point requires two neighbors, so that points right next to the boundary need special treatment. In practice, we have achieved optimal stability properties by evolving these points with second-order stencils. Our implementation requires one further ingredient in order to handle the spurious radiation inherent to numerically generated initial data of BH binary systems; cf. [92]. In order to avoid contamination of our simulations by such spurious radiation being trapped inside our reflective boundary, we employ standard outgoing radiation boundary conditions at early times and only switch on our reflective condition at

$$
t_{\text {ref }}=R_{B}+\Delta t_{\text {pulse }}
$$

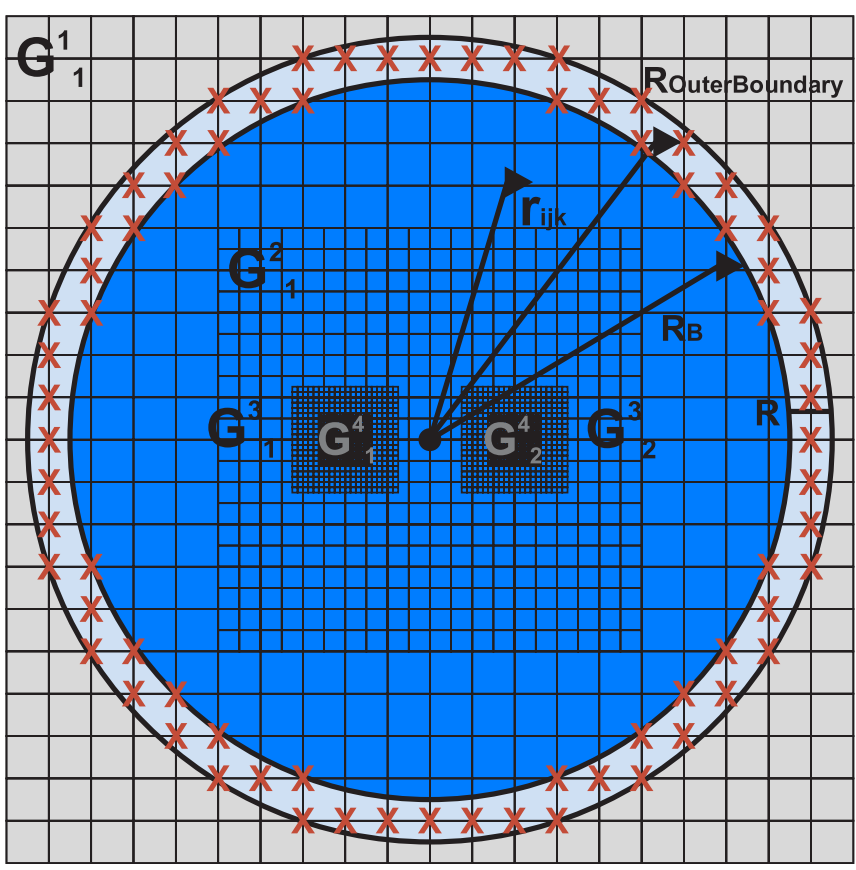

FIG. 2 (color online). Illustration of a (Lego-)spherical outer boundary. 
In order to avoid a discontinuous jump from outgoing to reflective boundary conditions, we gradually switch off the time derivative $\partial f / \partial t$ using a weighting factor $w(t)$ which smoothly decreases from 1 to 0 over an interval $\Delta t=10 M$ and $\Delta t=20 M$ for the head-on collision and inspiral, respectively. The duration of the spurious wave pulse $\Delta t_{\text {pulse }}$ is estimated from previous simulations of similar setups in asymptotically flat space-times as presented, for example, in Refs. $[76,93,94]$. The spurious radiation is thus given sufficient time to leave the computational domain.

\section{EXTRACTION OF MEANINGFUL QUANTITIES}

\section{A. Wave extraction}

Information about gravitational radiation is most commonly obtained in numerical simulations of $\mathrm{BH}$ binaries by computing the Weyl scalar $\Psi_{4}$, which has the asymptotic property of being equal to the outgoing radiation if the complex null tetrad is chosen properly. In a truly asymptotically AdS space-time, this procedure cannot be implemented so straightforwardly. In our toy model, which has the same local geometry as vacuum models, for a sufficiently large box size we expect the standard tetrad to yield the correct gravitational wave information. This is yet another advantage of our setup. Moreover, because we also deal with ingoing waves in our simulations, once they are reflected from the box boundary, we will consider the Weyl scalar $\Psi_{0}$ as well, in order to account for the ingoing contribution. The authors are not aware of any numerical study of the ingoing Weyl scalar $\Psi_{0}$. We will therefore analyze it in detail in the course of our numerical studies.

To be explicit, we define a spherical coordinate system centered on the center of mass of the binary with orthonormal basis $(\hat{r}, \hat{\theta}, \hat{\phi})$. The coordinates are chosen such that the azimuthal axis is aligned with the orbital angular momentum and the binary orbits are in the direction of increasing azimuthal coordinate. Our definitions and notation are the same as in $[76,95]$. To define our complex null tetrad, we use the timelike unit vector normal to a given hypersurface $\hat{n}$ and the radial unit vector $\hat{r}$ to define an ingoing $(\boldsymbol{k})$ and outgoing null vector $(\ell)$ by

$$
\boldsymbol{k} \equiv \frac{1}{\sqrt{2}}(\hat{n}+\hat{r}), \quad \ell \equiv \frac{1}{\sqrt{2}}(\hat{n}-\hat{r}) .
$$

We define the complex null vector $\boldsymbol{m}$ and its complex conjugate by

$$
\boldsymbol{m} \equiv \frac{1}{\sqrt{2}}(\hat{\phi}+i \hat{\theta}), \quad \overline{\boldsymbol{m}} \equiv \frac{1}{\sqrt{2}}(\hat{\phi}-i \hat{\theta}) .
$$

In terms of this tetrad, we define $\Psi_{0}$ and $\Psi_{4}$ as

$$
\begin{gathered}
\Psi_{0} \equiv C_{\alpha \beta \gamma \delta} k^{\alpha} m^{\beta} k^{\gamma} m^{\delta}, \\
\Psi_{4} \equiv C_{\alpha \beta \gamma \delta} \ell \bar{m}^{\beta} \ell \bar{m}^{\delta},
\end{gathered}
$$

where $C_{\alpha \beta \gamma \delta}$ is the Weyl tensor. To relate $\Psi_{0}$ and $\Psi_{4}$ to the amplitudes of the gravitational waves, we note that, in the transverse-traceless (TT) gauge, assuming the functional form $f(t \pm r)$ for the ingoing or outgoing waves, we have

$$
\begin{gathered}
\frac{1}{4}\left(\ddot{h}_{\hat{\theta} \hat{\theta}}^{T T}-\ddot{h}_{\hat{\phi} \hat{\phi}}^{T T}\right)=-R_{\hat{n} \hat{\theta} \hat{n} \hat{\theta}}=\mp R_{\hat{n} \hat{\phi} \hat{r} \hat{\phi}}=-R_{\hat{r} \hat{\theta} \hat{r} \hat{\theta}} \\
=R_{\hat{n} \hat{\phi} \hat{n} \hat{\phi}}= \pm R_{\hat{n} \hat{\theta} \hat{r} \hat{\theta}}=R_{\hat{r} \hat{\phi} \hat{r} \hat{\phi}}, \\
\frac{1}{2} \ddot{h}_{\hat{\theta} \hat{\phi}}^{T T}=-R_{\hat{n} \hat{\theta} \hat{n} \hat{\phi}}=-R_{\hat{r} \hat{\theta} \hat{r} \hat{\phi}}= \pm R_{\hat{n} \hat{\theta} \hat{r} \hat{\phi}}= \pm R_{\hat{r} \hat{\theta} \hat{n} \hat{\phi}} .
\end{gathered}
$$

A "dot" denotes derivative with respect to the argument. Following standard conventions, we take the $h_{+}$and $h_{\times}$polarizations of the gravitational waves to be given by

$$
\ddot{h}_{+}=\frac{1}{2}\left(\ddot{h}_{\hat{\theta} \hat{\theta}}^{T T}-\ddot{h}_{\hat{\phi} \hat{\phi}}^{T T}\right), \quad \ddot{h}_{\times}=\ddot{h}_{\hat{\theta} \hat{\phi}}^{T T} .
$$

Then, we find that in vacuum regions of the space-time, for outgoing waves $\ddot{h}_{+}=\ddot{h}_{+}(t-r)$ and $\ddot{h}_{\times}=\ddot{h}_{\times}(t-r)$,

$$
\begin{gathered}
\Psi_{0}=0, \\
\Psi_{4}=\ddot{h}_{+}+i \ddot{h}_{\times},
\end{gathered}
$$

while for ingoing waves $\ddot{h}_{+}=\ddot{h}_{+}(t+r)$ and $\ddot{h}_{\times}=$ $\ddot{h}_{\times}(t+r)$,

$$
\begin{gathered}
\Psi_{0}=\ddot{h}_{+}-i \ddot{h}_{\times}, \\
\Psi_{4}=0 .
\end{gathered}
$$

The fact that $\Psi_{4}\left(\Psi_{0}\right)$ are zero for ingoing (outgoing) waves is consistent with the leading order in perturbation theory. The correct expression and in particular its dependence on the radial coordinate are given by the solution of the Teukolsky master equation at large distances from the source, which states that for outgoing waves [96],

$$
\Psi_{0} \approx \frac{e^{i \omega(t-r)}}{r^{5}}, \quad \Psi_{4} \approx \frac{e^{i \omega(t-r)}}{r},
$$

while for ingoing waves,

$$
\Psi_{0} \approx \frac{e^{i \omega(t+r)}}{r}, \quad \Psi_{4} \approx \frac{e^{i \omega(t+r)}}{r^{5}} .
$$

We decompose the resulting $\Psi_{4}\left(\Psi_{0}\right)$ into modes by projection onto spherical harmonics of spin-weight $s=$ $-2(s=2)$ according to

$$
\begin{gathered}
M r \Psi_{4}=M r \sum_{l=2}^{\infty} \sum_{m=-l}^{l}{ }_{2} Y_{l m}(\theta, \phi) \psi_{l m}^{4}, \\
M r \Psi_{0}=M r \sum_{l=2}^{\infty} \sum_{m=-l}^{l}{ }_{2} Y_{l m}(\theta, \phi) \psi_{l m}^{0},
\end{gathered}
$$

where ${ }_{-2} Y_{l m}(\theta, \phi)$ and ${ }_{2} Y_{l m}(\theta, \phi)$ are spin-weight -2 and 2 spherical harmonics $[97,98]$. These are defined as 


$$
{ }_{s} Y_{l m}(\theta, \phi) \equiv(-1)^{s} \sqrt{\frac{2 l+1}{4 \pi}} d_{m(-s)}^{l}(\theta) e^{i m \phi},
$$

where $d_{m s}^{l}$ is the Wigner $d$-function

$$
\begin{aligned}
d_{m s}^{l}(\theta) \equiv & \sum_{t=C_{1}}^{C_{2}} \frac{(-1)^{t} \sqrt{(l+m) !(l-m) !(l+s) !(l-s) !}}{(l+m-t) !(l-s-t) ! t !(t+s-m) !} \\
& \times(\cos \theta / 2)^{2 l+m-s-2 t}(\sin \theta / 2)^{2 t+s-m},
\end{aligned}
$$

and where $C_{1}=\max (0, m-s)$ and $C_{2}=\min (l+m$, $l-s)$. Here $M$ is the ADM mass of the system, computed from the initial data and assuming this is an asymptotically flat space-time, and $r$ is the generalized harmonic radial coordinate.

In the numerical code, the null tetrad is constructed from a Cartesian orthonormal triad $(u, v, w)$ and the timelike vector $\hat{n}$ is orthonormal to $t=$ constant hypersurfaces. The space-time is evolved with time $t$ using Cartesian coordinates $x, y, z$. In practice, we compute the NewmanPenrose scalars $\Psi_{0}$ and $\Psi_{4}$ using the electromagnetic decomposition of the Weyl tensor according to the equations in (A11) on the entire Cartesian grid. Then, they are interpolated onto coordinate spheres of different extraction radii $r_{\mathrm{ex}}$ with a uniform distribution of points in $(\theta, \phi) .^{3}$ All the waveform-related data from the simulations presented in the course of this paper are taken from such samplings of $\Psi_{0}\left(t, r=r_{\mathrm{ex}}, \theta, \phi\right)$ and $\Psi_{4}(t, r=$ $\left.r_{\mathrm{ex}}, \theta, \phi\right)$. A more detailed description is given in Appendix A.

As discussed previously, $\Psi_{4}$ is no longer simply related to the energy flux, but since we are dealing with fairly large box sizes, one might hope that many notions can be retained in an approximate sense. Given the NewmanPenrose scalar $\Psi_{4}$, we can compute the radiated energy and linear and angular momentum from the radiation content [99]:

$$
\begin{array}{r}
\frac{d E}{d t}=\lim _{r \rightarrow \infty} \frac{r^{2}}{16 \pi} \int_{\Omega}\left|\int_{-\infty}^{t} \Psi_{4} d \tilde{t}\right|^{2} d \Omega, \\
\frac{d P_{i}}{d t}=-\lim _{r \rightarrow \infty} \frac{r^{2}}{16 \pi} \int_{\Omega} \ell_{i}\left|\int_{-\infty}^{t} \Psi_{4} d \tilde{t}\right|^{2} d \Omega, \\
\frac{d J_{z}}{d t}=-\lim _{r \rightarrow \infty} \frac{r^{2}}{16 \pi} \times \operatorname{Re}\left[\int_{\Omega}\left(\int_{-\infty}^{t} \Psi_{4} d \tilde{t}\right) \partial_{\phi}\right. \\
\left.\times\left(\int_{-\infty}^{t} \int_{-\infty}^{\hat{t}} \bar{\Psi}_{4} d \tilde{t} d \hat{t}\right) d \Omega\right],
\end{array}
$$

where

$$
l_{i}=(-\sin \theta \cos \phi, \quad-\sin \theta \sin \phi, \quad-\cos \theta) .
$$

\footnotetext{
${ }^{3} 108 \times 54$ points in $\theta \in[0, \pi], \phi \in[0,2 \pi]$ for the set of simulations using low resolution. In case of the higher resolutions, these numbers are adjusted accordingly.
}

The definitions above are based on time integrals which start in the infinite past (at retarded time $t=-\infty$ ), and thus capture the complete gravitational wave signal. Starting the time integrations at $t=-\infty$ corresponds to the limit of infinite extraction radius on the initial time slice-the slice would then extend all the way to spatial infinity, no part of the waveform would be lost, and it would take an infinite time for the waves to reach the extraction sphere. This situation cannot be handled with the current numerical codes; we therefore work with finite extraction radii.

The mass and angular momentum of the final $\mathrm{BH}$ can be estimated from balance arguments. Given the parameters $P_{y_{i}}, d$ in the Bowen-York initial data, we straightforwardly calculate the total initial angular momentum as

$$
J_{\text {ini }}=L_{\text {ini }}=d P_{y_{i}},
$$

since the initial spin of each BH is zero. Ansorg's TWOPUNCTURES [80] initial data solver directly provides the total ADM mass $M$ of the system, and we obtain radiated energy and angular momentum $E_{\text {rad }}$ and $J_{\text {rad }}$ from the gravitational wave signal. In case of a merger, this gives us the final angular momentum and mass of the BH:

$$
\begin{aligned}
& M_{\text {fin }}=M-E_{\text {rad }}, \\
& J_{\text {fin }}=J_{\text {ini }}-J_{\text {rad }} .
\end{aligned}
$$

The dimensionless spin parameter of the final hole follows directly from

$$
j_{\text {fin }}=\frac{J_{\text {fin }}}{M_{\text {fin }}^{2}} .
$$

We check our results by fitting the quasinormal frequency and damping time of the final $\mathrm{BH}$ and inverting them to obtain $j_{\mathrm{QNM}}$ (see e.g. [100-102]).

\section{B. Apparent horizon properties}

We can also characterize the process by the properties of the apparent horizon of the final BH itself. Since this relies only on local quantities, it does not depend upon the spacetime being asymptotically flat. In order to monitor the mass and spin of the final $\mathrm{BH}$, we use Thornburg's Apparent Horizon Finder AHFINDERDIRECT $[81,82]$ in different ways, which also allow us to obtain uncertainty estimates:

(1) The irreducible mass $M_{\text {irr }}$ enables us to calculate the final $\mathrm{BH}$ mass $M_{\mathrm{BH}}$ from Christodoulou's relation [103]:

$$
M_{\mathrm{BH}}^{2}=M_{\mathrm{irr}}^{2}+\frac{J^{2}}{4 M_{\mathrm{irr}}^{2}} .
$$

This relation provides a method to check the internal consistency of the result for the final $\mathrm{BH}$ spin as calculated from the above balance arguments. For 
this purpose we set $M_{\mathrm{BH}}=M_{\text {fin }}$ and solve Eq. (29) for the spin:

$$
j_{\text {fin }}^{2}=\frac{J^{2}}{M_{\text {fin }}^{2}}=4 \frac{M_{\mathrm{irr}}^{2}}{M_{\text {fin }}^{2}}\left(1-\frac{M_{\mathrm{irr}}^{2}}{M_{\mathrm{fin}}^{2}}\right) .
$$

For comparison we also compute the spin of the final hole from the two following estimates.

(2) We measure the ratio $C_{r}(j)=C_{p} / C_{e}$ of polar to equatorial circumference of the final $\mathrm{BH}$ [104]. If we assume the final object to be a Kerr $\mathrm{BH}$, this ratio is $C_{r}=\frac{2}{\pi} \sqrt{1-\beta^{2}} E\left(\beta^{2}\right)$, where $\beta^{2} \equiv j^{2} M /\left(2 r_{+}\right)$, $E\left(\beta^{2}\right)$ is a complete elliptic integral, and $r_{+} / M=$ $1+\sqrt{1-j^{2}}$. This expression can be inverted to find the dimensionless spin parameter, $j_{C_{r}}$, of the final hole.

(3) The equatorial circumference of a Kerr BH is $C_{e}=$ $4 \pi M$. Therefore, $2 \pi A_{\mathrm{AH}} / C_{e}^{2}=1+\sqrt{1-j_{\mathrm{AH}}^{2}}$, where $A_{\mathrm{AH}}$ is the area of the apparent horizon. Thus, the $\mathrm{AH}$ area and the equatorial circumference can be used to estimate the spin of the final $\mathrm{BH}$ from [105]:

$$
j_{\mathrm{AH}}=\sqrt{1-\left(\frac{2 \pi A_{\mathrm{AH}}}{C_{e}^{2}}-1\right)^{2}} .
$$

\section{NUMERICAL RESULTS}

Our numerical study focuses on two types of binary BH initial configurations; (i) head-on collisions of nonspinning BHs starting from rest and (ii) quasicircular inspiral of nonspinning holes. In the remainder of this work we label these as HD and IN simulations. The initial parameters of all our simulations as well as the structure of the computational domain and the position of the outer boundary
$R_{B}$ are summarized in Table I. Unless denoted otherwise, the results presented refer to the highest resolution available.

\section{A. Numerical convergence analysis}

Before we discuss in detail the physical properties of the BH binary systems, we test the accuracy of our simulations by performing a convergence analysis of model IN2. Numerical simulations based on the finite differencing method typically approximate the continuum solution of differential equations with a leading error that has a polynomial dependence on the resolution, $f_{\text {cont }}=f_{\text {num }}+$ $\mathcal{O}\left(h^{n}\right)$. The order $n$ depends on the specific numerical implementation. Consistency of the code is tested by evolving the same configurations with low, medium and high resolution $h_{c}, h_{m}$ and $h_{f}$. One straightforwardly shows that the convergence factor is then given by

$$
Q \equiv \frac{f_{h_{c}}-f_{h_{m}}}{f_{h_{m}}-f_{h_{f}}}=\frac{h_{c}^{n}-h_{m}^{n}}{h_{m}^{n}-h_{f}^{n}}
$$

where $f_{h}$ stands for any of the evolved variables obtained for resolution $h$. For the case of contemporary moving puncture codes, the solution is complicated by the fact that the differential equations are typically discretized with fourth (or higher) order accurate stencils, but prolongation in time between different refinement levels and implementation of outer boundary conditions is only second-order accurate; see Sec. IV in Ref. [107] and our discussion in Sec. II.

Figure 3 shows our convergence results for the variables $\Psi_{4}$ and $\Psi_{0}$ obtained for resolutions $h_{c}=M / 48, h_{m}=$ $M / 52$ and $h_{f}=M / 56$. Here the differences $f_{h_{m}}-f_{h_{f}}$ have been amplified by the convergence factors $Q=$ 1.26 and 1.47 expected for second- and fourth-order convergence, respectively. The figure indicates fourth-order accuracy for the first passage of the wave pulse and a gradual deterioration of convergence to second-order accuracy afterwards. We believe this to be a consequence

TABLE I. Grid structure, as well as initial and final parameters of the simulated black holes. The grid setup is given in terms of the radii of the individual refinement levels as well as the resolution near the punctures $h$ (see Sec. II E in [76] for details). The reflective outer boundary is located at radius $R_{B}$. The table further shows the initial coordinate separation of the two punctures $d$, the irreducible mass $M_{\text {irr }}$ and the Bowen-York [106] parameter for initial linear momentum $P_{i}$ of the individual holes. $J_{\text {fin }}^{\mathrm{AH}}$ and $J_{\text {fin }}^{\mathrm{rad}}$ are the spin of the single hole after merger determined from the AH and the merger radiation, respectively. All parameters are given in units of the ADM mass. We did not monitor the AH properties for all runs; therefore the spin of the final BH is not determined (empty cells) for some

\begin{tabular}{|c|c|c|c|c|c|c|c|}
\hline Run & Grid Setup & $R_{B} / M$ & $d / M$ & $M_{\mathrm{irr}, i} / M$ & $P_{i} / M$ & $J_{\text {fin }}^{\mathrm{AH}} / M^{2}$ & $J_{\text {fin }}^{\mathrm{rad}} / M^{2}$ \\
\hline IN1 & $\{(48,24,12,6) \times(1.5,0.75), h=1 / 56\}$ & 40 & 6.517 & 0.483 & \pm 0.133 & 0.69 & 0.70 \\
\hline IN2.1 & $\{(48,24,12,6) \times(1.5,0.75), h=1 / 48\}$ & 30 & 6.517 & 0.483 & \pm 0.133 & 0.69 & 0.65 \\
\hline IN2.2 & $\{(48,24,12,6) \times(1.5,0.75), h=1 / 52\}$ & 30 & 6.517 & 0.483 & \pm 0.133 & & 0.65 \\
\hline IN2.3 & $\{(48,24,12,6) \times(1.5,0.75), h=1 / 56\}$ & 30 & 6.517 & 0.483 & \pm 0.133 & & 0.65 \\
\hline HD1 & $\{(48,24,12,6) \times(1.5,0.75), h=1 / 60\}$ & 40 & 6.517 & 0.483 & 0.0 & 0.0 & 0.0 \\
\hline VIS & $\{(48,24,12,6) \times(1.5,0.75), h=1 / 48\}$ & 48 & 6.517 & 0.483 & \pm 0.133 & & \\
\hline
\end{tabular}
cases. The Weyl scalars have been extracted at $r_{\mathrm{ex}}=35 M$ (IN1, HD1) and $r_{\mathrm{ex}}=25 M$ (IN2), respectively. Model VIS is used in Fig. 9 in Appendix B for visualization. 

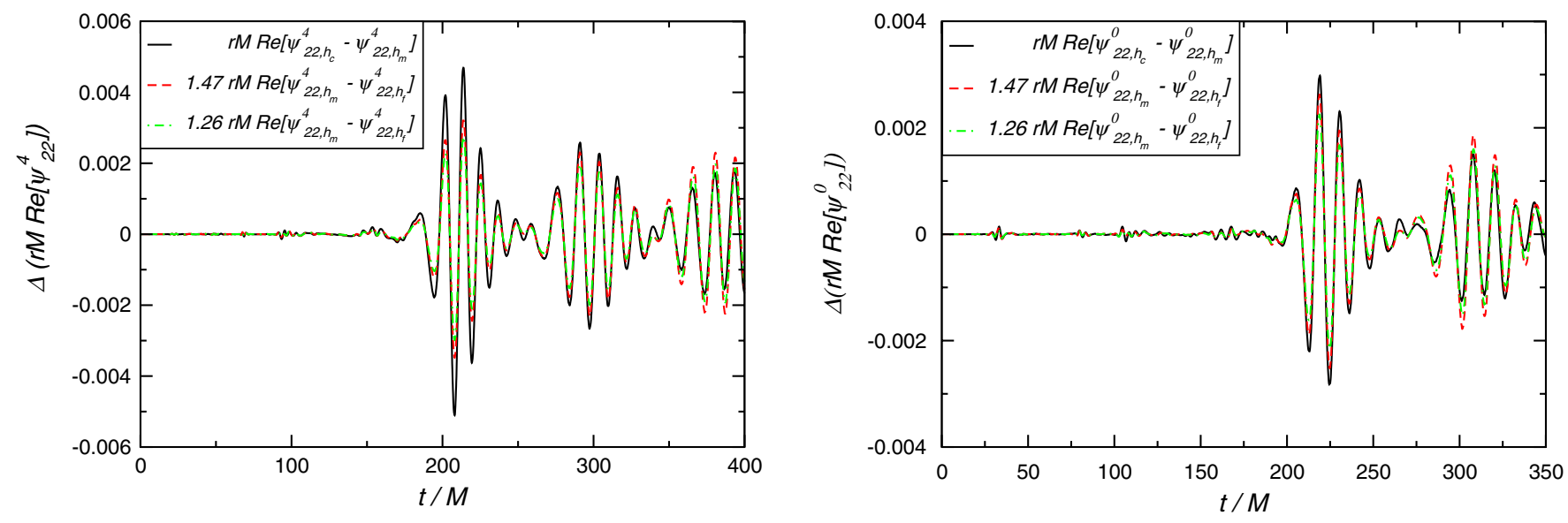

FIG. 3 (color online). Convergence analysis of the outgoing Weyl scalar $\Psi_{4}$ (left panel) and the ingoing Weyl scalar $\Psi_{0}$ (right panel) for the IN2 runs. We show the differences of the $l=m=2$ mode between the coarse and medium and the medium and fine resolution run. The latter has been amplified by the factors $Q=1.47$ (fourth-order convergence) and $Q=1.26$ (second-order convergence). We observe fourth-order convergence in the signal due to the merger whereas the first and second after-merger cycles show only secondorder convergence. The first two reflected and ingoing wave pulses show second-order convergence.

of the different ingredients of the code as discussed above. At early stages, the dominant error is the discretization of derivatives. As the pulse successively passes across mesh refinement boundaries and is reflected off the outer boundary, however, the second-order error in the prolongation operation becomes dominant and reduces the order of convergence. We also note, in this context, that well-posedness of the BSSN evolution system with reflective boundary condition has so far not been demonstrated. ${ }^{4}$ We therefore cannot rule out adverse effects on the longterm convergence properties due to potential ill-posedness of the continuum system of equations. In the remainder of this discussion, we will restrict ourselves to 2-3 passages of the wavepulse as covered in Fig. 3 during which the relative uncertainties in $\Psi_{4}$ and $\Psi_{0}$ are $\leq 5 \%$.

\section{B. Gravitational wave signal and black hole dynamics}

To our knowledge, this work presents the first analysis of gravitational waveforms with both outgoing $\left(\Psi_{4}\right)$ and ingoing $\left(\Psi_{0}\right)$ contributions for long-term stable numerical simulations of $\mathrm{BH}$ binaries. For this reason, we first illustrate the general pattern of the wave signal obtained for model VIS of Table I. A series of snapshots of both Newman-Penrose scalars are shown in Fig. 9 in Appendix B in superposed form.

The gravitational wave signal is dominated by the quadrupole contributions which we show in Fig. 4. For clarity,

\footnotetext{
${ }^{4}$ To our knowledge, the well-posedness of the system of equations in combination with reflecting boundary conditions, as treated here, has not been studied yet. Some investigations of the wave equation with this type of boundary conditions suggest that it may be ill-posed [108,109]. These investigations also show that the wave equation with periodic boundary conditions is a well-posed initial boundary value problem [108,109], pointing toward interesting future extension of our work.
}

the ingoing signal $\psi_{22}^{0}$ has been shifted in time by $\Delta t=$ $10 M$ in order to compensate for the additional propagation time from the extraction radius $r_{\mathrm{ex}}=35 \mathrm{M}$ to the boundary $R_{B}=40 M$ and back after reflection. The reflection introduces an additional phase shift of $\Delta \phi=\pi$ which has also been taken into account in the figure. Within numerical errors, we find the resulting outgoing and subsequent ingoing pulses to overlap.

The first outgoing wave pulse, visible in Fig. 4 around $150 \leq t / M \leq 250$, is generated during the inspiral, plunge and merger of the binary and is similar to waveforms obtained for the inspiral of nonspinning $\mathrm{BH}$ binaries in asymptotically flat space-times (cf. Fig. 1 in Refs. [76,107]). Because of the reflecting boundary,

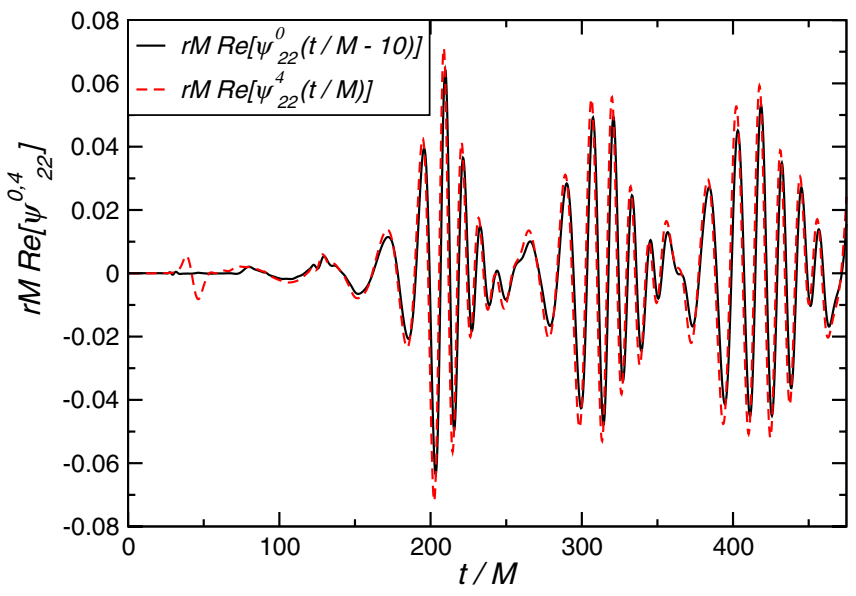

FIG. 4 (color online). Real part of the $l=m=2$ mode of $r M \Psi_{0}$ and $r M \Psi_{4}$ of run IN1. The ingoing signal $r M \Psi_{0}$ has been shifted in time by $\Delta t=10 \mathrm{M}$ and in phase by $\pi$ (thus equivalent to an extra minus sign) to account for the additional propagation time and the reflection. 

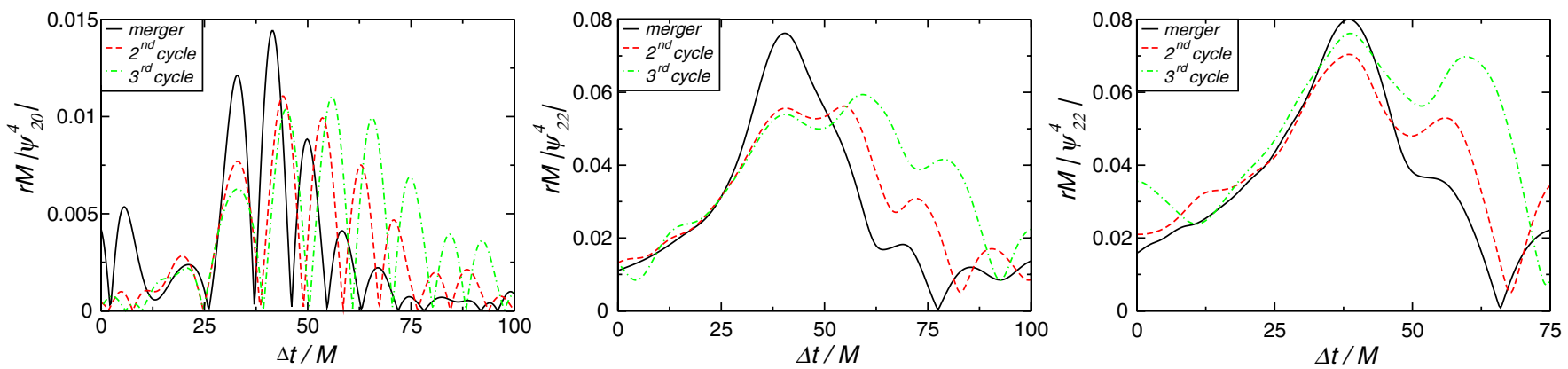

FIG. 5 (color online). Overlap of the amplitudes of successive pulses of the same waveform; $l=2, m=0$ for the HD1 run (left), $l=m=2$ for the IN1 ( center), and IN2.3 (right panel) simulations, obtained by time-shifting such that the maxima overlap.

however, this wave pulse does not escape the computational domain. Instead it propagates inwards, interacts with the post-merger remnant hole and eventually manifests itself as a second wave pulse shifted by $\Delta t \approx 80 \mathrm{M}$ relative to the first. This process repeats itself many times, with the wave pulse being presumably distorted (by absorption, superradiance and other curved space-time effects on wave propagation) upon each interaction with the $\mathrm{BH}$. We now investigate in detail these changes of the wave pulse upon interaction with the $\mathrm{BH}$.

\section{Interaction of the wave pulse with the remnant black hole}

As shown in Fig. 4, the outgoing and subsequent ingoing wave pulses overlap within numerical uncertainties. We therefore focus on the outgoing signal in our study of subsequent wave pulses and the gradual changes caused by successive scattering off the $\mathrm{BH}$. Changes in the wave pulse are best illustrated by considering the wave amplitude as shown in Fig. 5. Here we superpose the $l=2$, $m=0$ mode for model HD1 and the $l=2, m=2$ multipoles for models IN1 and IN2.3 of the first three successive outgoing wave pulses by applying corresponding time shifts to the waveform. Clearly, the wave pulses broaden after each scattering off the $\mathrm{BH}$. We emphasize that this distortion of the pulse is not an artifact of the outer boundary condition as is demonstrated by the good overlap between the ingoing and outgoing pulses in Fig. 4.

One possible explanation for this distortion relies on strong field, curved space-time effects: massless waves in curved geometries do not propagate on the light cone. Indeed, as shown in the classical work by DeWitt and Brehme, the Green's function for a massless field in a curved space-time does not generally vanish inside the light cone [110]. This gives rise to interesting effects. It is well known that wave propagation in $\mathrm{BH}$ space-times shows that the signal can roughly be divided into three parts: (i) The first part is the prompt response, at very early times, whose form depends strongly on the initial conditions. This is the most intuitive phase, being a counterpart of the light-cone propagation in flat space-time; (ii) At intermediate times the signal is dominated by an exponentially decaying ringing phase and corresponds to the excitation of the BH's characteristic modes of vibration [102]; (iii) A late-time tail, usually a power law falloff of the field [111,112]. Therefore, a variety of possible distortions is possible.

Another possible explanation for the increasing width of the pulse arises in the context of the superradiance mechanism. It is well known that the scattering of a wave pulse off a rotating Kerr BH will result in superradiant scattering - amplification of the scattered wave packet via extraction of rotational energy from the $\mathrm{BH}$-if the wave pulse satisfies

$$
\omega<m \Omega \text {, }
$$

where $\Omega \equiv j_{\text {fin }} /\left(2 r_{+}\right)$is the $\mathrm{BH}$ angular velocity $[69,113,114]$. We note, however, that Fig. 5 also indicates a broadening of scattered wave pulses in the head-on case where no superradiance is expected. While the observed broadening would be compatible with superradiance,

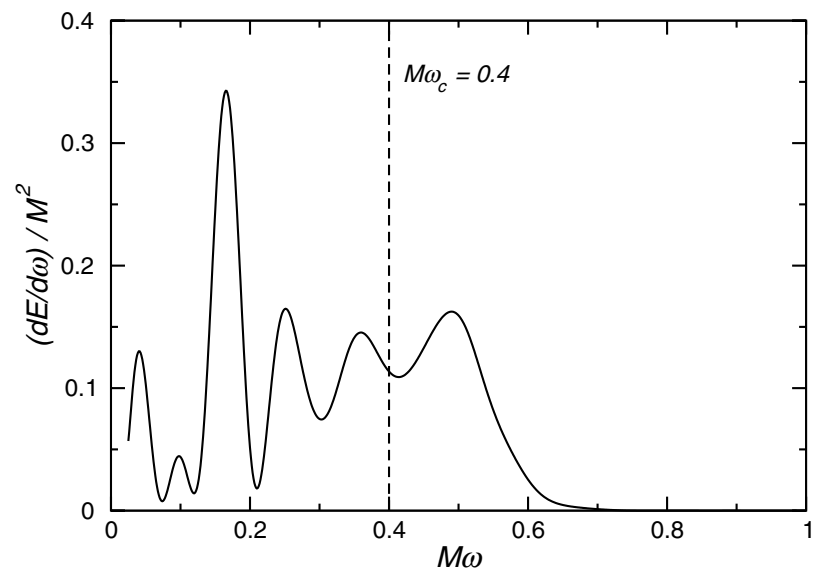

FIG. 6 (color online). The energy spectrum for the $l=m=2$ multipole of the outgoing scalar $\Psi_{4}$, for model IN2.3. This spectrum corresponds solely to the initial wave packet, i.e., the waveform has been truncated immediately before the first reflection off the boundary. The vertical line marks the threshold frequency for superradiance. 
other effects appear to also influence the shape of the pulse, and our observations do not conclusively demonstrate its presence.

In order to investigate this dispersion in more detail, we plot in Fig. 6 the energy spectrum for the dominant $l=2$, $m=2$ mode. The vertical line in this figure denotes the threshold frequency $m \Omega \approx 0.4 / M$ corresponding to a final spin $j_{\text {fin }}=0.69$ as obtained for the post-merger hole for configuration IN2; cf. Sec. III B. The figure demonstrates that the $l=2, m=2$ mode does contain contributions which would be subject to superradiance-induced amplification. These results then suggest that the low-frequency component of the pulse is amplified due to superradiance, while the high-frequency component is absorbed. A linear analysis of superradiance in the Kerr geometry [114] shows that superradiant effects are always small, unless the hole is rotating close to the extremal value. Thus, further studies, including larger spins of the post-merger hole, are necessary to comprehensively demonstrate superradiant wave amplification.

\section{Black hole dynamics}

In contrast to the case of asymptotically flat spacetimes, a $\mathrm{BH}$ binary under the influence of a reflective shell does not settle down into a stationary configuration soon after merger. This is due to the repeated interaction with the wave pulse passing back and forth across the spatially finite space-time. The prolonged dynamical state of the system manifests itself prominently in the area $A_{\mathrm{AH}}$ of the apparent horizon. In Fig. 7 we show the fractional deviation $\left(A_{\mathrm{AH}}-A_{0}\right) / A_{0}$ of the apparent horizon area from its value $A_{0}$ immediately after merger, i.e., the first instance a common apparent horizon is found. As expected, the horizon area remains nearly constant for the duration of the first passage of the pulse to the reflective shell and back, $\Delta t \approx 80 M$ and $60 M$, respectively, for models IN1 and IN2.1. ${ }^{5}$ The subsequent increase in $A_{\mathrm{AH}}$ demonstrates that some fraction of the gravitational wave pulse energy is absorbed by the hole. It follows another period of approximately constant horizon area, a further increase upon the second scattering of the pulse, and so on. Unfortunately, the radiation efficiency is almost 2 orders of magnitude lower for head-on collisions (see Table II in Ref. [76]), so that the increase in horizon area is buried in the numerical uncertainties. The headon case serves as a useful comparison, however, as it demonstrates that the changes observed for the inspiralling configurations are significant relative to numerical uncertainties.

The $\mathrm{BH}$ mass, defined in terms of the equatorial radius of the horizon $C_{e}$ by [105]

\footnotetext{
${ }^{5}$ Small oscillations in the horizon area are due to numerical uncertainties.
}

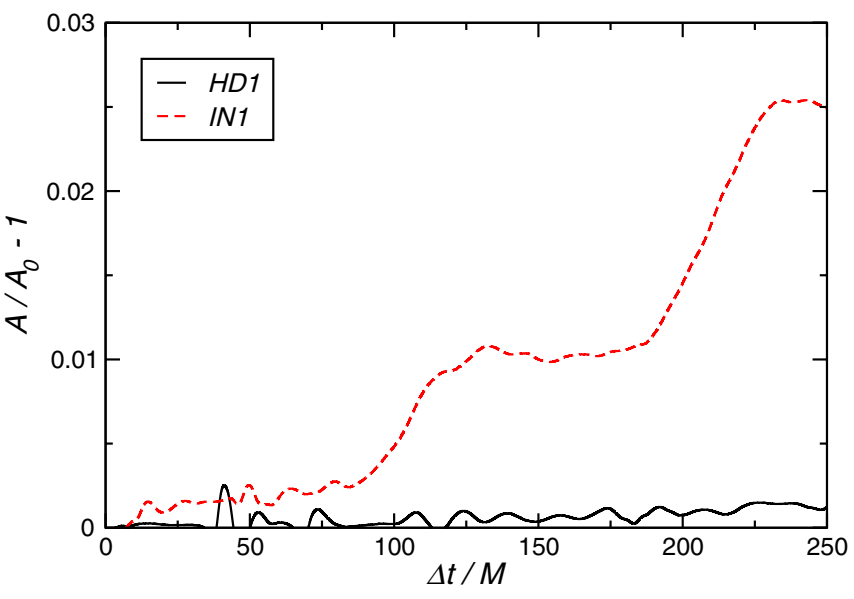

FIG. 7 (color online). Time evolution of the area of the apparent horizon for the head-on and inspiral simulation HD1 and IN1. The area of the rotating BH increases at regular intervals corresponding to the propagation time of the pulse between the hole and the reflective boundary at $r_{\mathrm{ex}}=35 \mathrm{M}$. Because of the small amount of radiation generated during the plunge in the head-on case (HD1), the variation in the AH area is buried in numerical noise.

$$
M=\frac{C_{e}}{4 \pi}
$$

shows a similar behavior as the horizon area. In Fig. 8 we plot the fractional deviation $\left(M-M_{0}\right) / M_{0}$ of the mass from its value immediately after merger together with the irreducible mass and the BH spin $J$ of the hole obtained for model IN1. The mass remains approximately constant until the pulse returns after its first reflection, then increases, remains constant during the second passage of the pulse, and so on. In contrast, the spin shows a significant increase only during the first scattering of the pulse off the $\mathrm{BH}$.

We conclude that, in each interaction with the gravitational radiation, the final $\mathrm{BH}$ mass increases. It is interesting

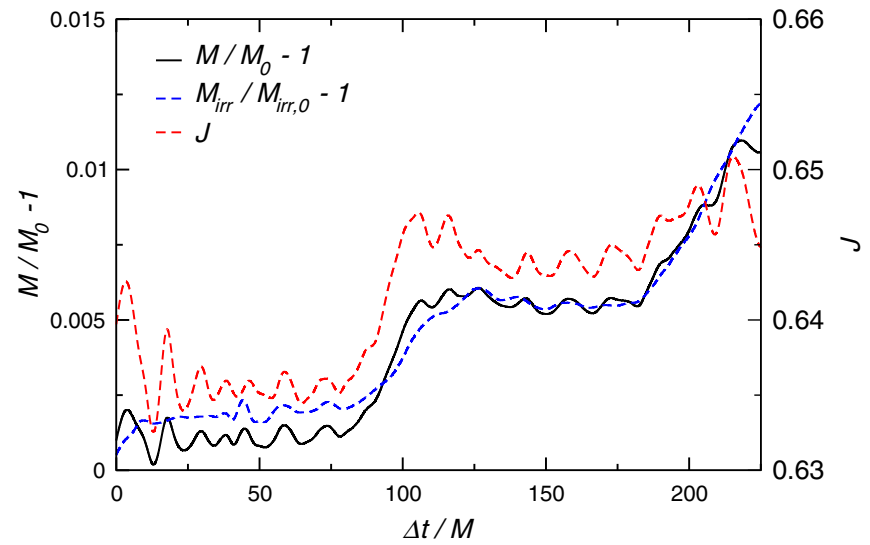

FIG. 8 (color online). Time evolution of the (relative) mass of the $\mathrm{BH}$ (solid line) computed by $M=C_{e} / 4 \pi$, the irreducible mass (dashed line) and the total spin $J=j M^{2}$ (dashed-dotted line). 
to compare the increase in the horizon mass with the amount of gravitational wave energy radiated during the last stages of the inspiral, plunge, and merger of a corresponding binary system in an asymptotically flat space-time which is about $3.5 \%$ of the total energy of the system [76,94]. For the IN1 run, we estimate that about $15 \%$ of the energy emitted during the merger is absorbed by the central spinning $\mathrm{BH}$ per interaction. Our results are consistent with total energy conservation. Moreover, they are not incompatible with superradiant amplification: typically, absorption of highfrequency waves is more effective than superradiant amplification of low-frequency waves, such that the net effect leads typically to absorption by the $\mathrm{BH}$ [114]. The prolonged nonstationary character of the post-merger state is also demonstrated by the time dependence of the BH's final spin. Immediately after merger, we obtain $j_{\text {fin }}=0.69$ from Eq. (31) in excellent agreement with corresponding simulations using outgoing radiation boundary conditions [94]. Successive interaction with the reflected wave pulse, however, results in a small but significant increase in the BH's spin as shown in Fig. 8. As before, the first increase occurs about $\Delta t=80 \mathrm{M}$ after merger, when the pulse has returned to the $\mathrm{BH}$. We estimate the fractional increase in spin resulting from the first scattering at about 5\%. For comparison, the total angular momentum radiated in the case of an asymptotically flat space-time is reported as $J^{\mathrm{rad}} / M^{2}=$ 0.246 (28\% of the initial orbital angular momentum of the system) in Table I of Ref. [94]. It thus appears that a significant amount of angular momentum remains in the form of gravitational waves. Because of numerical uncertainties it is not entirely clear whether later periods of interaction between pulse and hole result in a further transfer of angular momentum from the wave pulse to the hole or vice versa. Our results indicate, however, that the amount of angular momentum exchanged in subsequent interactions is significantly below $5 \%$.

\section{DISCUSSION}

The dynamics of BHs in generic space-times is a fascinating yet extremely challenging problem. The gauge/ gravity duality, however, strongly motivates us to solve dynamical problems with BHs on asymptotically AdS backgrounds. In this paper, we have studied a toy model that captures one of the fundamental features of such backgrounds: the active role played by the boundary conditions for the bulk evolution.

We have mimicked the global structure of an AdS background by introducing a reflecting wall at some radius. Within this cavity we evolved an inspiralling BH binary and a $\mathrm{BH}$ binary starting from rest at a certain initial distance. Of course, these are very specific initial configurations and serve merely as tests for future, possibly more complex, situations.

Perhaps the most important conclusion of the present work is that these simulations can be done and represent the first step to a full numerical evolution of $\mathrm{BHs}$ in $\mathrm{AdS}$ space-times. Indeed, as observed in Sec. IVA, it is not known whether the BSSN evolution scheme together with reflecting boundary conditions is a well-posed initial value problem. Thus, the convergence we have exhibited, which holds up to two reflections off the boundary of the gravitational radiation produced in the merger, is the first of our results. Among the other results presented here we stress the following:

(1) For the first time, we were able to numerically study the scalar $\Psi_{0}$, describing ingoing waves, and check in the numerical data the simple relations between $\Psi_{0}$ and $\Psi_{4}$ [cf. Eqs (12), (14), and (16) and Fig. 4].

(2) Our results are consistent with the intuitive expectations for a wave packet of radiation (generated during inspiral plus merger) travelling back and forth between the mirrorlike wall and the $\mathrm{BH}$ : part of this radiation is absorbed when interacting with the $\mathrm{BH}$ (especially high frequencies). We estimate that about $15 \%$ of the wave packet's energy is absorbed by the $\mathrm{BH}$ per interaction, at least during the first cycles.

(3) The wave packet is clearly distorted upon interaction with the hole, which means some frequencies are absorbed more efficiently than others. In principle, we should observe a small but nonzero superradiance. Unfortunately, we have not obtained incontrovertible proof of nonlinear superradiance from our numerical data. Presumably, the system will become unstable after a sufficiently long time, since the radiation should be exponentially amplified, once the high-frequency components had time to be completely absorbed by the BH. In future work we plan to investigate these instability studies further by considering a highly spinning, final $\mathrm{BH}$ produced by the inspiral of spinning BHs.

One issue that we have not explored in this paper is the potential influence of the box on the premerger dynamics. For sufficiently small boxes, it is plausible that the radiation produced in the inspiral may be reflected off the boundary and interact with the binary before the merger. This might produce observable signatures in the premerger dynamics and even in the properties of the remnant black hole. Whereas we seem to observe some hints of this effect in our numerical data, a more exhaustive analysis is required to produce some precise statements.

A future interesting extension of the present work is to repeat our analysis for periodic boundary conditions. Indeed, the wave equation in combination with periodic boundary conditions is a well-posed initial boundary value problem $[108,109]$. Such system is thus more likely to yield longer stable and convergent numerical evolutions, allowing one to address a number of interesting effects. Some relevant questions are: For how long is the numerical evolution stable? Do the numerical instabilities set in 
before the system has had time to reach a stable configuration? What are the typical times needed to achieve this?

Also in the context of numerical relativity in space-times with a cosmological constant, we plan on investigating dynamical black hole space-times in de Sitter backgrounds, where many of the problems present in AdS do not exist. (See also Refs. [115-117] for some work along these lines.)

Finally, it is necessary to extend this work in the obvious fashion: implement the evolution of BHs in real AdS backgrounds. We hope the present work will help in achieving that.

\section{ACKNOWLEDGMENTS}

We thank Leonardo Gualtieri, David Hilditch, and Florian Beyer for useful suggestions and discussions. We also thank the participants of the V Iberian Cosmology Meeting, the XII Marcel Grossmann Meetings, the Spanish Relativity Meeting 2009, and the I and II BH Workshop for useful feedback. M.Z. and H. W. are funded by Fundação para a Ciência e a Tecnologia (FCT) - Portugal through Grants No. SFRH/BD/43558/2008 and No. SFRH/ $\mathrm{BD} / 46061 / 2008$. A. N . is funded by FCT through Grant No. SFRH/BPD/47955/2008. This work was supported by the ERC Starting Grant No. DyBHo-256667, by Fundação Calouste Gulbenkian, by FCT-Portugal through Project Nos. CERN/FP/109306/2009 and CERN/FP/109290/ 2009, PTDC/FIS/64175/2006, PTDC/FIS/098025/2008, PTDC/FIS/098032/2008, PTDC/FIS/098962/2008 and PTDC/CTE-AST/098034/2008, by the Ramón y Cajal Programme of the Ministry of Education and Science of Spain, by NSF Grant No. PHY-0900735, and by the Fairchild Foundation of the California Institute of Technology. This research was supported by an allocation through the TeraGrid Advanced Support Program under Grant No. PHY-090003 and an allocation by the Centro de Supercomputación de Galicia (CESGA), Project No. ICTS-2009-40. Computations were performed on the TeraGrid clusters TACC Ranger and NICS Kraken, at Magerit in Madrid, Finis Terrae, the Milipeia cluster in Coimbra, the Woodhen cluster at Princeton University, and HLRB-II Garching. The authors thankfully acknowledge the computer resources, technical expertise, and assistance provided by the Barcelona Supercomputing Center (Centro Nacional de Supercomputación).

\section{APPENDIX A: ELECTROMAGNETIC DECOMPOSITION OF THE WEYL TENSOR}

Since in this work we analyze the Newman-Penrose scalar $\Psi_{0}$, besides $\Psi_{4}$, which is uncommon in numerical works, we collect in this appendix some useful results. Following the sign convention in $[76,95]$ the NewmanPenrose scalars $\Psi_{0}$ and $\Psi_{4}$ are computed by Eqs. (9) and (10). The vectors $\boldsymbol{k}, \boldsymbol{\ell}, \boldsymbol{m}, \overline{\boldsymbol{m}}$ form a null-tetrad. Their inner products vanish except for

$$
-\boldsymbol{k} \cdot \ell=1=\boldsymbol{m} \cdot \overline{\boldsymbol{m}} .
$$

In practice, the vectors of the null-tetrad are constructed from a Cartesian orthonormal basis $(u, v, w)$ in the spatial hypersurface and the timelike orthonormal vector $\hat{\boldsymbol{n}}$ according to

$$
\begin{array}{ll}
k^{\alpha}=\frac{1}{\sqrt{2}}\left(\hat{n}^{\alpha}+u^{\alpha}\right), & \ell^{\alpha}=\frac{1}{\sqrt{2}}\left(\hat{n}^{\alpha}-u^{\alpha}\right), \\
m^{\alpha}=\frac{1}{\sqrt{2}}\left(v^{\alpha}+i w^{\alpha}\right), & \bar{m}^{\alpha}=\frac{1}{\sqrt{2}}\left(v^{\alpha}-i w^{\alpha}\right) .
\end{array}
$$

The orthonormal triad vectors are constructed via the Gram-Schmidt orthonormalization, starting with

$$
\begin{gathered}
u^{i}=[x, y, z], \\
v^{i}=\left[x z, y z,-x^{2}-y^{2}\right], \\
w^{i}=\epsilon_{j k}^{i} u^{j} w^{k},
\end{gathered}
$$

where $\epsilon_{j k}^{i}$ is the three-dimensional Levi-Civita tensor. Next, we decompose the Weyl tensor in terms of its electric and magnetic parts [95]

$$
\begin{aligned}
C_{\alpha \beta \gamma \delta}= & 2\left(l_{\alpha[\gamma} E_{\delta] \beta}-l_{\beta[\gamma} E_{\delta] \alpha}-\hat{n}_{[\gamma} B_{\delta] \tau} \epsilon_{\alpha \beta}^{\tau}\right. \\
& \left.-\hat{n}_{[\alpha} B_{\beta] \tau} \epsilon_{\gamma \delta}^{\tau}\right),
\end{aligned}
$$

where $\quad l_{\mu \nu}=\gamma_{\mu \nu}+\hat{n}_{\mu} \hat{n}_{\nu} \quad$ and $\quad \epsilon_{\alpha \beta \gamma}=$ $\epsilon_{\mu \nu \lambda \rho} \hat{n}^{\mu} \perp_{\alpha}^{\nu} \perp_{\beta}^{\lambda} \perp_{\gamma}^{\rho}$. The electric and magnetic parts of the Weyl tensor are given by

$$
\begin{gathered}
E_{\alpha \beta}=C_{\mu \nu \lambda \rho} \perp_{\alpha}^{\mu} \hat{n}^{\nu} \perp_{\beta}^{\lambda} \hat{n}^{\rho}, \\
B_{\alpha \beta}={ }^{*} C_{\mu \nu \lambda \rho} \perp_{\alpha}^{\mu} \hat{n}^{\nu} \perp_{\beta}^{\lambda} \hat{n}^{\rho} .
\end{gathered}
$$

$\perp_{\nu}^{\mu}$ denotes the projection operator onto the hypersurface and $*$ denotes the Hodge dual. By using the Gauss-Codazzi equations we express the electromagnetic components in terms of the " $3+1$ " variables [76]

$$
E_{i j}=R_{i j}-\gamma^{k l}\left(K_{i j} K_{k l}-K_{i k} K_{j l}\right)
$$

$$
B_{i j}=\gamma_{i k} \epsilon^{k l m} D_{l} K_{m j}
$$

If we insert Eq. (A6), the definition of the null-tetrad (A2), and the expressions (A9) and (A10) into the definition of the Newman-Penrose scalars (9) and (10), we obtain 

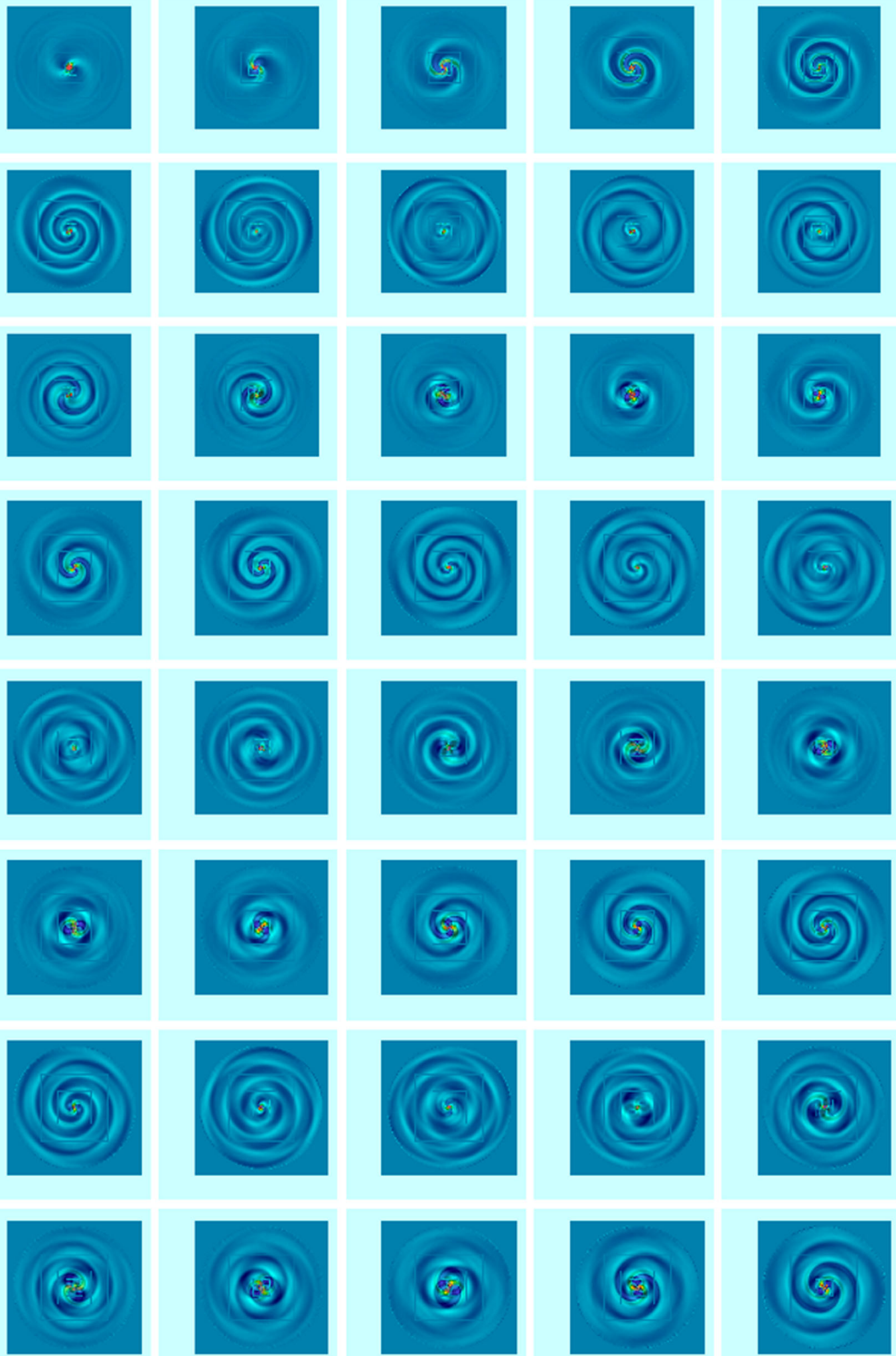

FIG. 9 (color online). Snapshots of $\mathfrak{R}\left(\Psi_{4}\right)$ superposed by $\mathfrak{R}\left(\Psi_{0}\right)$. The snapshots show the evolution from $t=150 M$ until $t=540 M$ and have a time interval of $\Delta t=10 \mathrm{M}$. We show a slice of the orbital plane with both coordinates going from $-48 M, \ldots, 48 M$. 


$$
\begin{aligned}
\Psi_{0}= & \frac{1}{2}\left[E_{k l}\left(v^{k} v^{l}-w^{k} w^{l}\right)+B_{k l}\left(v^{k} w^{l}+v^{l} w^{k}\right]\right. \\
& +\frac{i}{2}\left[E_{k l}\left(v^{k} w^{l}+v^{l} w^{k}\right)-B_{k l}\left(v^{k} v^{l}-w^{k} w^{l}\right)\right], \\
\Psi_{4}= & \frac{1}{2}\left[E_{k l}\left(v^{k} v^{l}-w^{k} w^{l}\right)-B_{k l}\left(v^{k} w^{l}+v^{l} w^{k}\right)\right] \\
& -\frac{i}{2}\left[E_{k l}\left(v^{k} w^{l}+v^{l} w^{k}\right)+B_{k l}\left(v^{k} v^{l}-w^{k} w^{l}\right)\right] .
\end{aligned}
$$

In the numerical code we use these relations in order to calculate $\Psi_{0}$ and $\Psi_{4}$ on the entire Cartesian grid. Then, they are interpolated onto coordinate spheres of various extraction radii $r_{\mathrm{ex}}$. The Newman-Penrose scalars $\Psi_{0}$ and $\Psi_{4}$ are decomposed into spin-weighted spherical harmonics ${ }_{s} Y_{l m}$ according to

$$
\begin{aligned}
\psi_{l m}^{0}(t) & =\int d \Omega \Psi_{0}\left(t, \theta, \phi_{2} Y_{l m}^{*} \theta, \phi\right) \\
& =(-)^{m+2} \int d \Omega \Psi_{0}(t, \theta, \phi)_{-2} Y_{l m}(\theta, \phi), \\
\psi_{l m}^{4}(t) & =\int d \Omega \Psi_{4}(t, \theta, \phi)_{-2} Y_{l m}^{*}(\theta, \phi) .
\end{aligned}
$$

In the first equation we have used the relation [97]

$$
{ }_{s} Y_{l m}^{*}=(-)^{m+s} Y_{l m} .
$$

Thus, in practice we implement $\psi_{l m}^{0}$ and $\psi_{l m}^{4}$ only in terms of the spherical harmonics ${ }_{-2} Y_{l m}$ with spin-weight -2 .

\section{APPENDIX B: SNAPSHOTS}

In Fig. 9 we illustrate the emission of the gravitational wave signal during the inspiral and merger and its evolution in the closed (confined) system containing a central, spinning $\mathrm{BH}$. We display snapshots of the waveforms by superposing (the real part of) $\Psi_{0}$ and $\Psi_{4}$ as obtained for model VIS of Table I. We show a slice of the orbital plane with $x, y=-48 M, \ldots, 48 M$ during an interval $t / M=$ $150, \ldots, 540$. The difference in time between the individual pictures is $\Delta t / M=10$. The series of snapshots starts in the late inspiral phase shortly before the plunge, and we see a strong gravitational wave signal that is emitted throughout the merger (first row and first two columns of the second row). This signal reaches the spherical boundary and is reflected back as can be seen in the final three panels of the second row and first two panels of the third row. Starting with the third snapshot in the third row we see a second pulse going outwards again after it has been scattered off the BH. This process is repeated several times and the series of snapshots ends with the fourth outgoing wave pulse. An animation constructed from the numerical data can be found in [118].
TABLE II. Parameters for a set of models evolved using a cubical boundary with a reflective boundary condition ("R b.c.") for models C1.1, C1.2 and C1.3 and an outgoing ("O b.c.") Sommerfeld condition for model O1.

\begin{tabular}{lcccc}
\hline \hline Run & Grid Setup & $h_{f} / M$ & $R_{B} / M$ & b.c. \\
\hline O1 & $(192,96,48,24,12,6)(1.5,0.75)$ & $1 / 40$ & 192 & $\mathrm{O}$ \\
C1.1 & $(24,12,6)(1.5,0.75)$ & $1 / 40$ & 24 & $\mathrm{R}$ \\
C1.2 & $(24,12,6)(1.5,0.75)$ & $1 / 44$ & 24 & $\mathrm{R}$ \\
C1.3 & $(24,12,6)(1.5,0.75)$ & $1 / 48$ & 24 & $\mathrm{R}$ \\
\hline \hline
\end{tabular}

\section{APPENDIX C: CUBIC-SHAPED BOX}

We have also performed simulations using condition (5) on a cubic outer boundary. These simulations represent the inspiral of nonspinning $\mathrm{BH}$ binaries with initial separation $d / M=6.514$ and initial linear momentum

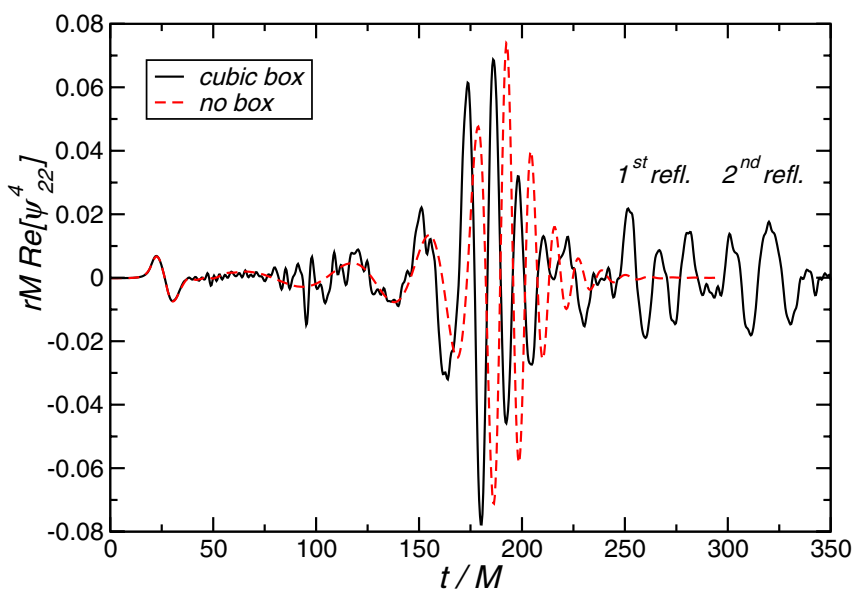

FIG. 10 (color online). Comparison of the $l=2, m=2$ modes of $\Psi_{4}$ obtained for models $\mathrm{C} 1.1$ (solid) and $\mathrm{O} 1$ (dashed curve). The expected ranges in time for subsequent wave pulses resulting from first and second reflections are indicated in the figure.

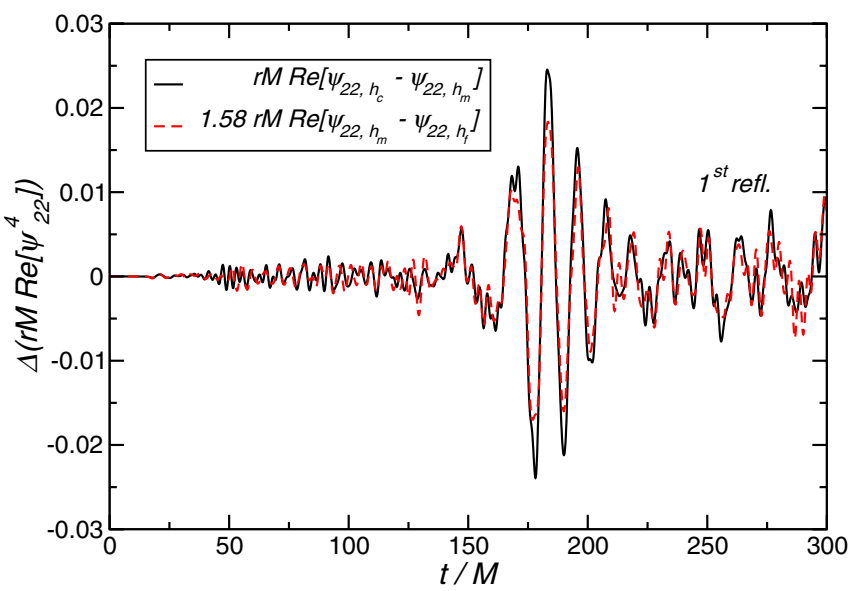

FIG. 11 (color online). Convergence analysis of the $l=m=2$ mode of $\Psi_{4}$ obtained for model C1 of Table II. 

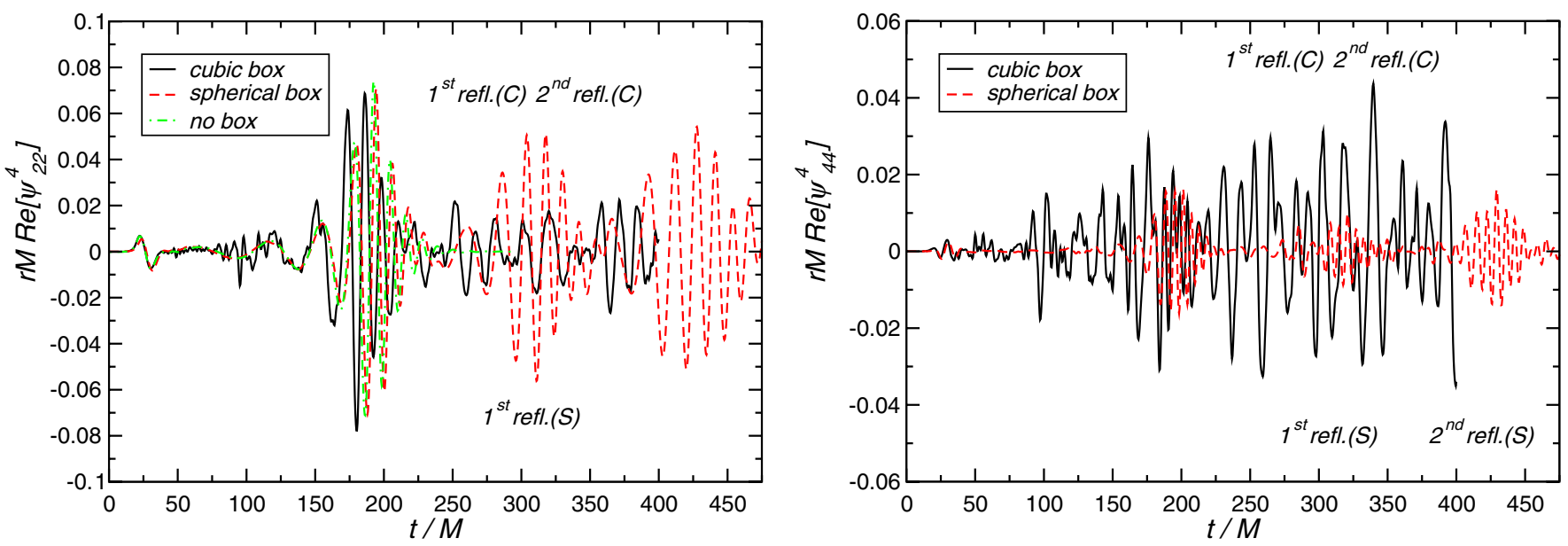

FIG. 12 (color online). Comparison of the $l=m=2$ (left) and $l=m=4$ (right) modes of $\Psi_{4}$ obtained for models C1.3 (cubic boundary), IN1 (spherical boundary), and O1 (outgoing condition).

$P_{y_{i}} / M= \pm 0.133$. The grid setup for these runs is listed in Table II together with a reference model $\mathrm{O} 1$ which describes the inspiral of the same binary in an asymptotically flat space-time using outgoing radiation boundary conditions. Gravitational waves have been extracted at $r_{\mathrm{ex}}=$ $20 M$ in the form of the Newman-Penrose scalar $\Psi_{4}$.

In Fig. 10, we compare the $l=m=2$ mode of $\Psi_{4}$ obtained by the evolution of models $\mathrm{C} 1.1$ and $\mathrm{O} 1$.

In Fig. 11 we present the convergence plot of the $l=$ $m=2$ mode of $\Psi_{4}$ obtained from evolutions of models C1.1, C1.2 and C1.3. The difference between the medium and fine resolution results has been amplified by the factor
$Q=1.58$, corresponding to fourth-order convergence. While the overall convergence is about fourth order as in the case of a spherical shell, the cubical outer boundary introduces a substantial amount of numerical noise, which is also demonstrated in Fig. 12 which displays the $l=2$, $m=2$ and the $l=4, m=4$ multipoles of $\Psi_{4}$ for models $\mathrm{C} 1.3$ and IN1. We believe that this is at least partly a consequence of mode mixing in the case of the cubical boundary shell which is not well-suited for the geometric shape of the gravitational wave pulse. For this reason, we have exclusively used a spherical shell in the main part of this work.
[1] F. Pretorius, Phys. Rev. Lett. 95, 121101 (2005).

[2] M. Campanelli, C. O. Lousto, P. Marronetti, and Y. Zlochower, Phys. Rev. Lett. 96, 111101 (2006).

[3] J. G. Baker, J. Centrella, D.-I. Choi, M. Koppitz, and J. van Meter, Phys. Rev. Lett. 96, 111102 (2006).

[4] M. Boyle et al., Phys. Rev. D 76, 124038 (2007).

[5] J. A. González, U. Sperhake, and B. Brügmann, Phys. Rev. D 79, 124006 (2009).

[6] C.O. Lousto, H. Nakano, Y. Zlochower, and M. Campanelli, Phys. Rev. Lett. 104, 211101 (2010).

[7] C. O. Lousto, H. Nakano, Y. Zlochower, and M. Campanelli, arXiv:1008.4360.

[8] C. O. Lousto and Y. Zlochower, arXiv:1009.0292.

[9] S. Dain, C. O. Lousto, and Y. Zlochower, Phys. Rev. D 78, 024039 (2008).

[10] I. Hinder, Classical Quantum Gravity 27, 114004 (2010).

[11] J. A. González, U. Sperhake, B. Brügmann, M. Hannam, and S. Husa, Phys. Rev. Lett. 98, 091101 (2007).

[12] J. A. González, M.D. Hannam, U. Sperhake, B. Brügmann, and S. Husa, Phys. Rev. Lett. 98, 231101 (2007).
[13] M. Campanelli, C. O. Lousto, Y. Zlochower, and D. Merritt, Phys. Rev. Lett. 98, 231102 (2007).

[14] P. Ajith et al., Classical Quantum Gravity 24, S689 (2007).

[15] P. Ajith et al., Phys. Rev. D 77, 104017 (2008).

[16] A. Buonanno et al., Phys. Rev. D 76, 104049 (2007).

[17] A. Buonanno et al., Phys. Rev. D 79, 124028 (2009).

[18] U. Sperhake, V. Cardoso, F. Pretorius, E. Berti, and J. A. González, Phys. Rev. Lett. 101, 161101 (2008).

[19] U. Sperhake et al., Phys. Rev. Lett. 103, 131102 (2009).

[20] M. Shibata, H. Okawa, and T. Yamamoto, Phys. Rev. D 78, 101501 (2008).

[21] F. Pretorius and D. Khurana, Classical Quantum Gravity 24, S83 (2007).

[22] J. M. Maldacena, Int. J. Theor. Phys. 38, 1113 (1999).

[23] A. Strominger and C. Vafa, Phys. Lett. B 379, 99 (1996).

[24] C. G. Callan and J. M. Maldacena, Nucl. Phys. B472, 591 (1996).

[25] E. Witten, Adv. Theor. Math. Phys. 2, 253 (1998).

[26] S. W. Hawking and D. N. Page, Commun. Math. Phys. 87, 577 (1983). 
[27] G. T. Horowitz and V. E. Hubeny, Phys. Rev. D 62, 024027 (2000).

[28] D. Birmingham, I. Sachs, and S. N. Solodukhin, Phys. Rev. Lett. 88, 151301 (2002).

[29] P. Kovtun, D. T. Son, and A. O. Starinets, Phys. Rev. Lett. 94, 111601 (2005)

[30] M. W. Choptuik, Phys. Rev. Lett. 70, 9 (1993).

[31] L. Alvarez-Gaume, C. Gomez, and M. A. Vazquez-Mozo, Phys. Lett. B 649, 478 (2007).

[32] R. M. Wald, J. Math. Phys. (N.Y.) 21, 2802 (1980).

[33] A. Ishibashi and R. M. Wald, Classical Quantum Gravity 20, 3815 (2003).

[34] A. Ishibashi and R. M. Wald, Classical Quantum Gravity 21, 2981 (2004).

[35] D. T. Son and A. O. Starinets, J. High Energy Phys. 09 (2002) 042.

[36] C.P. Herzog and D. T. Son, J. High Energy Phys. 03 (2003) 046.

[37] K. Skenderis and B. C. van Rees, Phys. Rev. Lett. 101, 081601 (2008).

[38] K. Skenderis and B. C. van Rees, J. High Energy Phys. 05 (2009) 085.

[39] M. Zilhao et al., Phys. Rev. D 81, 084052 (2010).

[40] H. Witek et al., Phys. Rev. D 82, 104014 (2010).

[41] H. Yoshino and M. Shibata, Phys. Rev. D 80, 084025 (2009).

[42] E. Sorkin, Phys. Rev. D 81, 084062 (2010).

[43] E. Sorkin and M. W. Choptuik, Gen. Relativ. Gravit. 42, 1239 (2009).

[44] K.-i. Nakao, H. Abe, H. Yoshino, and M. Shibata, Phys. Rev. D 80, 084028 (2009).

[45] M. Shibata and H. Yoshino, Phys. Rev. D 81, 021501 (2010).

[46] L. Lehner and F. Pretorius, Phys. Rev. Lett. 105, 101102 (2010).

[47] S. W. Hawking and H. S. Reall, Phys. Rev. D 61, 024014 (1999).

[48] V. Cardoso and O. J.C. Dias, Phys. Rev. D 70, 084011 (2004).

[49] V. Cardoso, O. J. C. Dias, J. P. S. Lemos, and S. Yoshida, Phys. Rev. D 70, 044039 (2004).

[50] V. Cardoso, O. J. C. Dias, and S. Yoshida, Phys. Rev. D 74, 044008 (2006).

[51] H. Kodama, R. A. Konoplya, and A. Zhidenko, Phys. Rev. D 79, 044003 (2009).

[52] K. Murata, Prog. Theor. Phys. 121, 1099 (2009).

[53] H. Kodama, Prog. Theor. Phys. Suppl. 172, 11 (2008).

[54] A. N. Aliev and O. Delice, Phys. Rev. D 79, 024013 (2009).

[55] N. Uchikata, S. Yoshida, and T. Futamase, Phys. Rev. D 80, 084020 (2009).

[56] V. Cardoso and O. J.C. Dias, J. High Energy Phys. 04 (2009) 125.

[57] V. Cardoso, O. J. C. Dias, and J. V. Rocha, J. High Energy Phys. 01 (2010) 021.

[58] S. Hollands, A. Ishibashi, and R. M. Wald, Commun. Math. Phys. 271, 699 (2007).

[59] S. Hollands and A. Ishibashi, Commun. Math. Phys. 291, 443 (2009).

[60] W.H. Press and S. A. Teukolsky, Nature (London) 238, 211 (1972).

[61] T. Damour, N. Deruelle, and R. Ruffini, Lett. Nuovo Cimento 15, 257 (1976).
[62] S. Detweiler, Phys. Rev. D 22, 2323 (1980).

[63] T. J. M. Zouros and D. M. Eardley, Ann. Phys. (N.Y.) 118, 139 (1979).

[64] H. Furuhashi and Y. Nambu, Prog. Theor. Phys. 112, 983 (2004).

[65] M. J. Strafuss and G. Khanna, Phys. Rev. D 71, 024034 (2005).

[66] S. Hod and O. Hod, Phys. Rev. D 81, 061502 (2010).

[67] J. G. Rosa, J. High Energy Phys. 06 (2010) 015.

[68] V. Cardoso and J.P. S. Lemos, Phys. Lett. B 621, 219 (2005).

[69] V. Cardoso and S. Yoshida, J. High Energy Phys. 07 (2005) 009.

[70] M. H. P. M. van Putten, Science 284, 115 (1999).

[71] A. N. Aguirre, Astrophys. J. 529, L9 (2000).

[72] R. Arnowitt, S. Deser, and C. W. Misner, arXiv:gr-qc/ 0405109.

[73] J. W. York, Jr., in Sources of Gravitational Radiation, edited by L.L. Smarr (Cambridge University Press, Cambridge, England, 1979), pp. 83-126.

[74] M. Shibata and T. Nakamura, Phys. Rev. D 52, 5428 (1995).

[75] T. W. Baumgarte and S. L. Shapiro, Phys. Rev. D 59, 024007 (1998).

[76] U. Sperhake, Phys. Rev. D 76, 104015 (2007).

[77] CACTUS Computational Toolkit, http://www.cactuscode .org/.

[78] E. Schnetter, S.H. Hawley, and I. Hawke, Classical Quantum Gravity 21, 1465 (2004).

[79] Mesh refinement with CARPET, http://www.carpetcode.org/

[80] M. Ansorg, B. Brügmann, and W. Tichy, Phys. Rev. D 70, 064011 (2004).

[81] J. Thornburg, Phys. Rev. D 54, 4899 (1996).

[82] J. Thornburg, Classical Quantum Gravity 21, 743 (2004).

[83] U. Sperhake et al., Phys. Rev. D 78, 064069 (2008).

[84] M. Alcubierre et al., Phys. Rev. D 67, 084023 (2003).

[85] D. Pollney, C. Reisswig, E. Schnetter, N. Dorband, and P. Diener, arXiv:0910.3803.

[86] D. Pollney, C. Reisswig, N. Dorband, E. Schnetter, and P. Diener, Phys. Rev. D 80, 121502 (2009).

[87] C. Reisswig, N. T. Bishop, D. Pollney, and B. Szilagyi, Phys. Rev. Lett. 103, 221101 (2009).

[88] C. Reisswig, N. T. Bishop, D. Pollney, and B. Szilagyi, Classical Quantum Gravity 27, 075014 (2010).

[89] O. Rinne, L. Lindblom, and M. A. Scheel, Classical Quantum Gravity 24, 4053 (2007).

[90] E. Pazos, M. Tiglio, M. D. Duez, L. E. Kidder, and S. A. Teukolsky, Phys. Rev. D 80, 024027 (2009).

[91] D. Shoemaker et al., Classical Quantum Gravity 20, 3729 (2003).

[92] T. Bode, D. Shoemaker, F. Herrmann, and I. Hinder, Phys. Rev. D 77, 044027 (2008).

[93] J. G. Baker, J. Centrella, D.-I. Choi, M. Koppitz, and J. van Meter, Phys. Rev. D 73, 104002 (2006).

[94] E. Berti et al., Phys. Rev. D 76, 064034 (2007).

[95] H. Friedrich, Classical Quantum Gravity 13, 1451 (1996).

[96] S. A. Teukolsky, Astrophys. J. 185, 635 (1973).

[97] J. N. Goldberg, A. J. MacFarlane, E. T. Newman, F. Rohrlich, and E. C. G. Sudarshan, J. Math. Phys. (N.Y.) 8, 2155 (1967).

[98] E. Berti, V. Cardoso, and M. Casals, Phys. Rev. D 73, 024013 (2006). 
[99] M. Alcubierre, Introduction to $3+1$ Numerical Relativity, International Series of Monographs on Physics Vol. 140 (Oxford University Press, New York, 2008).

[100] E. Berti, V. Cardoso, and C. M. Will, Phys. Rev. D 73, 064030 (2006).

[101] E. Berti, V. Cardoso, J. A. González, and U. Sperhake, Phys. Rev. D 75, 124017 (2007).

[102] E. Berti, V. Cardoso, and A. O. Starinets, Classical Quantum Gravity 26, 163001 (2009).

[103] D. Christodoulou, Phys. Rev. Lett. 25, 1596 (1970).

[104] P. Anninos et al., Phys. Rev. D 50, 3801 (1994).

[105] K. Kiuchi, Y. Sekiguchi, M. Shibata, and K. Taniguchi, Phys. Rev. D 80, 064037 (2009).

[106] J. M. Bowen and J. W. York Jr., Phys. Rev. D 21, 2047 (1980).

[107] B. Brügmann et al., Phys. Rev. D 77, 024027 (2008).

[108] B. Gustafsson, H.O. Kreiss, and J. Oliger, Time Dependent Problems and Difference Methods (Wiley, New York, 1995).
[109] D. Hilditch (private communication).

[110] B. S. DeWitt and R. W. Brehme, Ann. Phys. (N.Y.) 9, 220 (1960).

[111] E. S. C. Ching, P. T. Leung, W. M. Suen, and K. Young, Phys. Rev. D 52, 2118 (1995).

[112] V. Cardoso, S. Yoshida, O. J.C. Dias, and J.P. S. Lemos, Phys. Rev. D 68, 061503 (2003).

[113] J.M. Bardeen, W.H. Press, and S.A. Teukolsky, Astrophys. J. 178, 347 (1972).

[114] S. A. Teukolsky and W. H. Press, Astrophys. J. 193, 443 (1974).

[115] F. Beyer, Ph.D. thesis, Max Planck Institute for Gravitational Physics and University of Potsdam, 2007, arXiv:0710.4297.

[116] F. Beyer, Classical Quantum Gravity 25, 235005 (2008).

[117] F. Beyer, J. Comput. Phys. 228, 6496 (2009).

[118] http://blackholes.ist.utl.pt/. 